\title{
A critical appraisal of NLO+PS matching methods
}

\author{
Stefan Höche, ${ }^{a}$ Frank Krauss, ${ }^{b}$ Marek Schönherr ${ }^{b}$ and Frank Siegert ${ }^{c}$ \\ ${ }^{a}$ SLAC National Accelerator Laboratory, \\ Menlo Park, CA 94025, U.S.A. \\ ${ }^{b}$ Institute for Particle Physics Phenomenology, Durham University, \\ Durham DH1 3LE, U.K. \\ ${ }^{c}$ Physikalisches Institut, Albert-Ludwigs-Universität Freiburg, \\ D-79104 Freiburg, Germany \\ E-mail: shoeche@slac.stanford.edu, frank.krauss@durham.ac.uk, \\ marek.schoenherr@durham.ac.uk, frank.siegert@cern.ch
}

ABSTRACT: In this publication, uncertainties in and differences between the MC@NLO and POWHEG methods for matching next-to-leading order QCD calculations with parton showers are discussed. Implementations of both algorithms within the event generator SHERPA and based on Catani-Seymour subtraction are employed to assess the impact on a representative selection of observables. In the case of MC@NLO a substantial simplification is achieved by using dipole subtraction terms to generate the first emission. A phase space restriction is employed, which allows to vary in a transparent way the amount of nonsingular radiative corrections that are exponentiated. Effects on various observables are investigated, using the production of a Higgs boson in gluon fusion, with or without an associated jet, as a benchmark process. The case of $H+$ jet production is presented for the first time in an NLO+PS matched simulation. Uncertainties due to scale choices and non-perturbative effects are explored in the production of $W^{ \pm}$and $Z$ bosons in association with a jet. Corresponding results are compared to data from the Tevatron and LHC experiments.

KeYwords: QCD Phenomenology

ARXIV EPRINT: 1111.1220 


\section{Contents}

1 Introduction 1

2 Event generation with Powheg and MC@Nlo in a nutshell 3

2.1 Anatomy of NLO calculations 3

2.2 Sudakov form factors 4

2.3 Matrix element corrections and PowHEG 4

2.4 Modified subtraction and MC@NLO 5

2.5 Subtleties in practical implementations of POWHEG and MC@NLO 6

3 Powheg and MC@Nlo in detail $\quad 8$

3.1 Notation and definitions 8

$\begin{array}{llr}3.2 & \text { From fixed-order to resummation } & 9\end{array}$

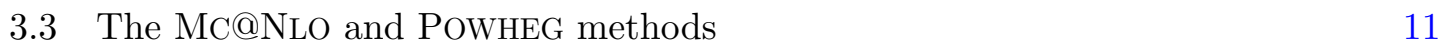

$\begin{array}{ll}3.4 & \text { Event-generation techniques } \\ \end{array}$

4 Analysis of perturbative uncertainties $\quad 14$

$\begin{array}{ll}4.1 \text { Higgs-boson production in gluon fusion } & 14\end{array}$

4.2 Higgs-boson production in association with a jet 21

$4.3 W$-boson production in association with a jet 25

5 Non-perturbative uncertainties and comparison to data 27

$\begin{array}{ll}5.1 & \text { Analysis of non-perturbative effects }\end{array}$

$5.2 Z+$ jet production compared to Tevatron data 30

$5.3 W+$ jet production compared to LHC data 34

6 Conclusions $\quad 34$

$\begin{array}{ll}\text { A Comment on the NLL accuracy of the PowHEg formula } & 37\end{array}$

\section{Introduction}

The central topic in the development of Monte-Carlo event generators during the past decade was the systematic inclusion of higher order QCD effects. One of the first approaches, the merging of leading-order (LO) multi-jet matrix elements of varying multiplicity with parton showers, has been pioneered and matured in a series of papers [1-10], such that by now it has become the accepted standard for simulating final states which include multi-jet topologies. It typically yields a very satisfactory description of experimental data, but due to the lack of virtual corrections it can never achieve the formal accuracy of a full next-to-leading order (NLO) calculation. This shortcoming is especially worrisome in the case of Standard Model Higgs-boson production through gluon fusion, where a large 
$K$-factor indicates substantial higher-order corrections, such that theoretical predictions should be made at next-to-leading order accuracy or better.

With the development of the MC@NLO [11] and PowHEG $[12,13]$ techniques it became feasible to combine NLO accurate matrix-element calculations with parton showers. This technology is called NLO matching, in contrast to the LO merging described above. Ultimately, NLO matching techniques allow to carry out a full simulation of events, including hadronisation, hadron decays and the underlying event. While MC@NLO relies on a subtraction algorithm based on the parton shower approximation to collinear divergences in real-emission matrix elements, the POWHEG method effectively constructs a one-emission generator, similar to a one-step parton shower, with evolution kernels determined by ratios of real-emission and Born matrix elements. In this respect the POWHEG method is very similar to traditional matrix-element correction techniques [14-17].

Despite having been proposed several years ago, the MC@NLO and POWHEG methods were not applied to processes which can become singular at Born level, such as di-jet production, until recently. This delay signals several complications which arise in reactions with additional light partons. Di-jet production [18] and $Z+j$ production [19] have now become available through the PowhEGBox [20] and, more recently, $W^{ \pm}$plus two jets production was implemented [21] using MC@NLO methods.

In this publication the MC@NLO and POWHEG methods will be compared in some detail, and open issues related to their implementation and validation will be discussed. As benchmark processes, the production of Higgs-, $W^{ \pm}$- and $Z$-bosons, alone or in association with one hard jet at Born level have been chosen. These processes are either signals central to the experimental program at the LHC, or they contribute as important backgrounds to many new physics searches [22-26]. Apart from that, QCD-associated $W^{ \pm}$- and $Z$ production are of great interest, to study jet production and evolution in a hadron-collider environment at the energy frontier [27-31], to improve the jet energy scale determination by the experiments, or to study multiple parton scattering processes. At the LHC as well as at the Tevatron, typically, good agreement is found when comparing respective data with merged leading-order predictions or with next-to-leading order perturbative QCD predictions, for instance from [32-39]. This level of theoretical control and experimental precision suggests that the processes chosen here are indeed well-suited to serve as testbed for NLO matching methods.

The present paper is organised as follows: In section 2 basic ideas underlying the MC@NLO and POWHEG strategies are reviewed. Similarities and differences between the two methods are discussed and potential pitfalls in their implementation are indicated. The solution to some of these problems is detailed in section 3, where the MC@NLO algorithm is worked out in a formalism similar to [40]. This allows to elaborate on various aspects of the method regarding non-trivial colour and flavour configurations. Readers not interested in the technical details may skip this section. In section 4 perturbative uncertainties related to scale variations and to the different exponentiation in the MC@NLO and POWHEG methods are highlighted. The impact of non-perturbative effects is investigated in section 5 and results for $W^{ \pm}$- and $Z$-production are compared to experimental data. Finally, section 6 summarises the findings of this publication and outlines some possible extensions of the methods presented here. 


\section{Event generation with PowHEG and MC@NLO in a nutshell}

This section is meant to highlight in a simple language the ideas of how NLO QCD calculations can be combined with subsequent parton showers. A common notation is established for POWHEG and MC@NLO and critical aspects related to the practical implementation for processes with non-trivial colour structure are discussed.

\subsection{Anatomy of NLO calculations}

In order to see how the existing matching algorithms work, consider first the structure of an NLO calculation. The total cross section can be written as

$$
\sigma^{(\mathrm{NLO})}=\int \mathrm{d} \Phi_{B}\left[\mathrm{~B}\left(\Phi_{B}\right)+\tilde{\mathrm{V}}\left(\Phi_{B}\right)+\mathrm{I}^{(\mathrm{S})}\left(\Phi_{B}\right)\right]+\int \mathrm{d} \Phi_{R}\left[\mathrm{R}\left(\Phi_{R}\right)-\mathrm{D}^{(\mathrm{S})}\left(\Phi_{R}\right)\right],
$$

where $\mathrm{d} \Phi_{B}$ and $\mathrm{d} \Phi_{R}$ denote Born and real-emission phase space, respectively and $\mathrm{B}, \tilde{\mathrm{V}}$, $\mathrm{I}^{(\mathrm{S})}, \mathrm{R}$ and $\mathrm{D}^{(\mathrm{S})}$, are the matrix elements for the Born, virtual, integrated subtraction, real emission and real subtraction contribution. Note that for hadronic initial states $\tilde{V}$ is defined such as to include the collinear mass-factorisation counterterms. All integrands include parton luminosity and flux factors. For simplicity it is assumed that the processes under consideration here have no identical QCD partons in the final state, a detailed discussion of symmetry factors is postponed to section 3. In the subtraction formalisms most commonly employed to date [41-44], subtraction terms can be written as the convolution of Born matrix elements with suitably defined operators $\tilde{K}$, such that schematically

$$
\mathrm{d} \Phi_{R} \mathrm{D}^{(\mathrm{S})}\left(\Phi_{R}\right)=\mathrm{d} \Phi_{B} \mathrm{~d} \Phi_{1}\left[\mathrm{~B}\left(\Phi_{B}\right) \otimes \tilde{\mathrm{K}}\left(\Phi_{1}\right)\right] \text { and } \mathrm{I}^{(\mathrm{S})}\left(\Phi_{B}\right)=\int \mathrm{d} \Phi_{1}\left[\mathrm{~B}\left(\Phi_{B}\right) \otimes \tilde{\mathrm{K}}\left(\Phi_{1}\right)\right],
$$

indicating that the two related contributions cancel locally in the Born phase space. This also suggests that the definition of the subtraction kernels $\tilde{\mathrm{K}}$ is somewhat arbitrary, as long as they exhibit the same singularity structure as the full real-emission matrix elements in the soft and collinear limits.

To combine an NLO calculation with a parton shower, it is essential to ensure that the result inherits the total cross section from the fixed-order calculation. This could trivially be achieved by multiplying a leading order plus parton shower simulation with a local $K$-factor - the ratio of NLO and LO cross section. The problem is that parton showers generate emissions which do not necessarily follow the pattern obtained from the real emission matrix elements, leading to a mismatch of radiation patterns at first order in the strong coupling constant $\alpha_{s}$. In principle, this problem has been solved by the traditional matrix-element correction procedure outlined in [14-17], which is implemented for a number of processes in both HERWIG and PYTHIA. The essence of this method lies in the fact that, for the processes it is applied to, the product of Born-level contribution and parton shower evolution kernel $\mathrm{K}$ is larger than the corresponding real emission term $\mathrm{R}$ in the complete phase space of the extra emission. This allows to correct the first (hardest) emission with a factor R/(B K), leading to the desired distribution in phase space. From this it can be seen that such an algorithm will fail for cases, where $\mathrm{R}$ is not always smaller than the Born-times-parton-shower result, or where the parton shower is not capable of filling the full phase space. In such cases a more detailed analysis is necessary. 


\subsection{Sudakov form factors}

In this section the construction of the parton shower using Sudakov form factors will be briefly recalled. They are defined as

$$
\Delta\left(t, t^{\prime}\right)=\exp \left\{-\int_{t}^{t^{\prime}} \frac{\mathrm{d} \bar{t}}{\bar{t}} \int \mathrm{d} z \int \frac{\mathrm{d} \phi}{2 \pi} J(\bar{t}, z, \phi) \frac{\alpha_{s}}{2 \pi} \mathrm{K}(\bar{t}, z, \phi)\right\}=\exp \left\{-\int_{t}^{t^{\prime}} \mathrm{d} \Phi_{1} \frac{\alpha_{s}}{2 \pi} \mathrm{K}\left(\Phi_{1}\right)\right\},
$$

where the sequence of emissions is ordered by a parameter $t$ - typically related to the virtuality, transverse momentum or opening angle of the emission. Furthermore, $z$ is the variable defining the splitting of energy or light-cone momentum of the two daughters emerging in the decay of the parton, and $\phi$ denotes the azimuthal angle. Collectively they define the one-emission phase space $\Phi_{1}$ and its Jacobian $J$, while $\mathrm{K}$ is the splitting kernel encoding the detailed kinematical distribution due to soft-collinear enhancement and spin effects. For simplicity, parton luminosity factors are included into this kernel, and a detailed discussion is postponed to section 3 . Note that in all practical implementations of eq. (2.3) the argument of the strong coupling is related to the transverse momentum of the splitting, given by the decay kinematics, i.e. by $\Phi_{1}$. For the sake of clarity this argument will be suppressed in this section.

This Sudakov form factor can be interpreted as a no-branching probability between the two scales $t$ and $t^{\prime}$. Obviously, $1-\Delta\left(t, t^{\prime}\right)$ then yields the probability that a splitting has taken place in the interval between the two scales. Therefore, to first order in $\alpha_{s}$, the cross section in the parton-shower approximation reads

$$
\sigma_{\mathrm{PS}}^{(\mathrm{LO})}=\int \mathrm{d} \Phi_{B} \mathrm{~B}\left(\Phi_{B}\right)\left[\Delta\left(t_{0}, \mu_{F}^{2}\right)+\int_{t_{0}}^{\mu_{F}^{2}} \mathrm{~d} \Phi_{1} \frac{\alpha_{s}}{2 \pi} \mathrm{K}\left(\Phi_{1}\right) \Delta\left(t, \mu_{F}^{2}\right)\right],
$$

where $t$ is determined by the kinematics of the first emission, $t=t\left(\Phi_{1}\right)$, such that it is smaller than the suitably chosen, process-dependent factorisation scale $\mu_{F}^{2}$, and where the arguments of the splitting kernel depend on this extra emission kinematics. The first term in the square bracket represents the probability of no extra emission to happen above the infrared cut-off $t_{0}$ of the parton shower, while the second term generates one emission above this scale. It can also be seen that the square bracket as a whole integrates to one, exposing the probabilistic nature of the parton shower, which leaves the total cross section of an event sample unchanged.

\subsection{Matrix element corrections and PowHEG}

Applying matrix element corrections to the parton shower transforms the equation above, eq. (2.4), into

$$
\sigma_{\mathrm{MEC}}^{(\mathrm{LO})}=\int \mathrm{d} \Phi_{B} \mathrm{~B}\left(\Phi_{B}\right)\left[\bar{\Delta}\left(t_{0}, \mu_{F}^{2}\right)+\int_{t_{0}}^{\mu_{F}^{2}} \mathrm{~d} \Phi_{1} \frac{\mathrm{R}\left(\Phi_{B}, \Phi_{1}\right)}{\mathrm{B}\left(\Phi_{B}\right)} \bar{\Delta}\left(t, \mu_{F}^{2}\right)\right],
$$

where the modified Sudakov form factor $\bar{\Delta}$ reads

$$
\bar{\Delta}\left(t, t^{\prime}\right)=\exp \left\{-\int_{t}^{t^{\prime}} \mathrm{d} \Phi_{1} \frac{\mathrm{R}\left(\Phi_{B}, \Phi_{1}\right)}{\mathrm{B}\left(\Phi_{B}\right)}\right\} .
$$


Again, the square bracket in eq. (2.5) integrates to one, and the cross section of an event sample is identical to the Born cross section. However, the distribution of the first (hardest) emission, to first order in $\alpha_{s}$ will follow the pattern given by the full real emission matrix element. It is thus correct to $\mathrm{O}\left(\alpha_{s}\right)$ if the upper integration limit in the real-emission term is extended to the hadronic centre-of-mass energy. This corresponds to a powershower approach $[45,46]$ and ensures coverage of the full phase space. However, it also implies extending the resummation built into the parton shower beyond the region of its validity and is therefore unjustified. A more detailed discussion of this point can be found in appendix A. The problem can be solved for example in the MC@NLO method, see section 2.4 .

In order to achieve full $\mathrm{O}\left(\alpha_{s}\right)$-accuracy in both the cross section of the produced event sample and the pattern of the first emission it is mandatory to replace the differential Born cross section $\mathrm{d} \Phi_{B} \mathrm{~B}$ with an expression that integrates to the full NLO cross section of eq. (2.1). This is achieved by the substitution

$$
B\left(\Phi_{B}\right) \longrightarrow \overline{\mathrm{B}}\left(\Phi_{B}\right)=\mathrm{B}\left(\Phi_{B}\right)+\tilde{\mathrm{V}}\left(\Phi_{B}\right)+\mathrm{I}^{(\mathrm{S})}\left(\Phi_{B}\right)+\int \mathrm{d} \Phi_{1}\left[\mathrm{R}\left(\Phi_{B}, \Phi_{1}\right)-\mathrm{D}^{(\mathrm{S})}\left(\Phi_{B}, \Phi_{1}\right)\right]
$$

It is straightforward to interpret this term as an NLO-weighted Born-level cross section, or, put in a slightly different way, a Born-level cross section that is augmented with a local $K$-factor.

Equipped with this definition the expression for the cross section in the PowHEGformalism reads

$$
\sigma_{\text {POWHEG }}^{(\mathrm{NLO})}=\int \mathrm{d} \Phi_{B} \overline{\mathrm{B}}\left(\Phi_{B}\right)\left[\bar{\Delta}\left(t_{0}\right)+\int_{t_{0}} \mathrm{~d} \Phi_{1} \frac{\mathrm{R}\left(\Phi_{B}, \Phi_{1}\right)}{\mathrm{B}\left(\Phi_{B}\right)} \bar{\Delta}(t)\right] .
$$

This expression is accurate up to the first order in the strong coupling constant for both the total cross section of the event sample and the differential cross section with respect to the kinematics of the first emission.

\subsection{Modified subtraction and MC@NLO}

As indicated above, hard real-emission configurations should not be included in the exponentiation in eq. (2.8). There are also contributions which can generically not be obtained from a Born-level configuration by simply emitting an extra parton. Examples for this include flavour processes such as $u \bar{u} \rightarrow c \bar{s} e^{-} \bar{\nu}_{e}$ or kinematical configurations such as radiation zeroes [47-49]. Therefore, it is well motivated to split the real-emission matrix elements $\mathrm{R}$ into an infrared-singular (soft) and an infrared-regular (hard) part, $\mathrm{D}^{(\mathrm{A})}$ and $\mathrm{H}^{(\mathrm{A})}$, respectively: $\mathrm{R}=\mathrm{D}^{(\mathrm{A})}+\mathrm{H}^{(\mathrm{A})}[50]$. Equation (2.1) then transforms into

$$
\begin{aligned}
\sigma^{(\mathrm{NLO})}= & \int \mathrm{d} \Phi_{B}\left[\mathrm{~B}\left(\Phi_{B}\right)+\tilde{\mathrm{V}}\left(\Phi_{B}\right)+\mathrm{I}^{(\mathrm{S})}\left(\Phi_{B}\right)\right] \\
& +\int \mathrm{d} \Phi_{R}\left[\mathrm{D}^{(\mathrm{A})}\left(\Phi_{R}\right)-\mathrm{D}^{(\mathrm{S})}\left(\Phi_{R}\right)\right]+\int \mathrm{d} \Phi_{R} \mathrm{H}^{(\mathrm{A})}\left(\Phi_{R}\right)
\end{aligned}
$$


and the cross section, eq. (2.8), can be rewritten as

$$
\sigma_{\mathrm{MC} @ N L O}^{(\mathrm{NLO})}=\int \mathrm{d} \Phi_{B} \overline{\mathrm{B}}^{(\mathrm{A})}\left(\Phi_{B}\right)\left[\bar{\Delta}^{(\mathrm{A})}\left(t_{0}\right)+\int_{t_{0}} \mathrm{~d} \Phi_{1} \frac{\mathrm{D}^{(\mathrm{A})}\left(\Phi_{B}, \Phi_{1}\right)}{\mathrm{B}\left(\Phi_{B}\right)} \bar{\Delta}^{(\mathrm{A})}(t)\right]+\int \mathrm{d} \Phi_{R} \mathrm{H}^{(\mathrm{A})}\left(\Phi_{R}\right),
$$

where

$$
\overline{\mathrm{B}}^{(\mathrm{A})}\left(\Phi_{B}\right)=\mathrm{B}\left(\Phi_{B}\right)+\tilde{\mathrm{V}}\left(\Phi_{B}\right)+\mathrm{I}^{(\mathrm{S})}\left(\Phi_{B}\right)+\int \mathrm{d} \Phi_{1}\left[\mathrm{D}^{(\mathrm{A})}\left(\Phi_{B}, \Phi_{1}\right)-\mathrm{D}^{(\mathrm{S})}\left(\Phi_{B}, \Phi_{1}\right)\right]
$$

and, with obvious notation,

$$
\bar{\Delta}^{(\mathrm{A})}\left(t, t^{\prime}\right)=\exp \left\{-\int_{t}^{t^{\prime}} \mathrm{d} \Phi_{1} \frac{\mathrm{D}^{(\mathrm{A})}\left(\Phi_{B}, \Phi_{1}\right)}{\mathrm{B}\left(\Phi_{B}\right)}\right\} .
$$

In this manner, the non-singular contributions at real-emission level are correctly captured and their exponentiation can be avoided. This is how the MC@NLO formalism works [11].

\subsection{Subtleties in practical implementations of POWHEG and MC@NLO}

At the level of detail of the discussion up to now, an implementation of both methods, POWHEG and MC@NLO, seems quite straightforward. However, there are a number of subtleties, which have not been presented yet in a coherent fashion in a journal publication.

1. Subtraction of infrared divergent sub-leading colour terms in MC@NLO

The parton shower is based on a leading logarithmic and leading colour approximation. Using it, without modification, to define modified subtraction terms $\mathrm{D}^{(\mathrm{A})}$ will therefore necessarily miss divergences in sub-leading colour configurations and will result in infinite integration results in eq. (2.11). This presents a clear obstacle to apply the method to processes where infrared divergences in such sub-leading colour configurations emerge. In [51], and referring to ideas outlined in the original MC@NLO publication [11], this problem was overcome for the case of heavy flavour hadro-production processes of the type $g g \rightarrow Q \bar{Q}$ and $q \bar{q} \rightarrow Q \bar{Q}$ at Born-level. An additional factor was introduced, which multiplies the complete integrand in eq. (2.11) and tends to zero as emissions become soft. This same method is applied in the case of the recently presented aMc@NLO [52, 53].

We propose a different, exact and process-independent solution here, which relies on choosing $\mathrm{D}^{(\mathrm{A})}=\mathrm{D}^{(\mathrm{S})}$, leading to a tremendous simplification of eq. (2.11). In the remainder of this publication we will refer to this scheme as the MC@NLO method. Technical details of how $\mathrm{D}^{(\mathrm{S})}$ is exponentiated into a Sudakov form factor will be given in section 3 .

2. Choice of scales in Powheg and MC@NLO

It was argued in $[12,13]$, based on [54], that the proper choice of scale for the strong coupling in the kernel R/B of the POWHEG Sudakov form factor is given by the transverse momentum of the emission generated in the branching process. Similarly, it was 
pointed out in [11] that the scale in the evolution kernel of an MC@NLO should be the transverse momentum. This particular choice of scale effectively resums a certain class of universal higher-order corrections. It typically leads to a very good agreement between the results from existing parton shower algorithms and experimental data and is therefore employed in all standard Monte-Carlo event generators [55].

Such a scale choice ultimately implies higher-order corrections to the event kinematics. The key point is that the differential NLO cross section defined by eq. (2.7) can be evaluated with arbitrary scales. However, the functional form of the scale must be infrared and collinear safe. This is not the case for the transverse momentum in the branching process, because, in contrast to the Sudakov branching algorithm, there is no infrared cutoff in the calculation of $\overline{\mathrm{B}}$. Thus, the first-order expansion of the Sudakov form factor does not reproduce the exact same real-correction / subtraction terms as the NLO calculation. The differences are numerically large in the logarithmically enhanced regions of phase space, where it is not expected that PowHEG or MC@NLO reproduce the exact leading-order result. But even the region of hard radiation can be affected, depending on how much phase space is covered by $\mathrm{D}^{(\mathrm{A})}$.

3. Exponentiation of non-leading logarithms in POWHEG

Ultimately, there is a last subtlety, which has also been discussed in [20], summarising some previous work by the same authors. The evolution kernel R/B in the POWHEG method generates subleading logarithms and it is somewhat questionable in how far they should be exponentiated. The difference compared to evolution kernels in the parton shower may lead to sizable effects, up to orders of magnitude, when results for the hard, non-logarithmic tails of distributions in the POWHEG approach are compared with those of pure NLO calculations, see for instance [56].

In this context, it is important to distinguish two effects: One is exponentiation of $\mathrm{R} / \mathrm{B}$ beyond the factorization scale $\mu_{F}$ (cf. eq. (2.8)), the other is the difference in the functional form of the evolution kernels itself. The former effect can be emulated in our implementation of the MC@NLO formalism and we will examine it more closely in sections 4.1 and 4.2. We use CS subtraction and a parton shower built on CS subtraction kernels [57]. Initially the starting scales of the parton shower will be maximised for the first emission, in order to fill the full phase space, similar to the POWHEG method and a power-shower approach $[45,46]$. To show that the main difference between POWHEG and MC@NLO lies in the enlarged phase space in POWHEG, a simple phase-space constraint is added to the subtraction [58], limiting the logarithmically enhanced region of the real-emission contribution and adding the hard regions to the regular contribution. The impact of the choice of this constraint is analysed quantitatively in section 4 . The respective variations in the result are referred to as "exponentiation uncertainties". It is important to note that this uncertainty is minimised in the MC@NLO method, as the phase space constraint is determined by the resummation scale. In this publication we simply use the MC@NLO framework to make the problem in POWHEG explicit. 


\section{Powheg and MC@NLO in detail}

When combining next-to-leading order matrix elements with parton showers, the logarithmic corrections encoded in the Sudakov form factor must be matched to the full nextto-leading order prediction of the parton-level calculation. This is usually achieved either by subtracting the first-order expansion of the resummed result from the NLO calculation $\left(\mathrm{MC} @ \mathrm{NLO}\right.$ with $\left.\mathrm{D}^{(\mathrm{A})}=\mathrm{D}^{(\mathrm{S})}\right)$, or by exponentiating the full real-emission correction in the resummation (POWHEG). One can also construct mixed schemes, as argued in section 2.4. The difference which is induced by the exponentiation of subleading corrections vanishes for soft or collinear parton emission. However, it might play an important role in other regions of the phase space. In the following, a formalism that allows to discuss the difference between the MC@NLO and POWHEG methods in more detail is reviewed.

\subsection{Notation and definitions}

Denoting sets of $n$ particles in a $2 \rightarrow(n-2)$ process by $\{\vec{a}\}=\left\{a_{1}, \ldots, a_{n}\right\}$, while their respective flavours and momenta are specified separately through $\{\vec{f}\}=\left\{f_{1}, \ldots, f_{n}\right\}$ and $\{\vec{p}\}=\left\{p_{1}, \ldots, p_{n}\right\}$, the generic expression for a fully differential Born-level cross section can be written as a sum over all contributing flavour configurations:

$$
\mathrm{d} \sigma_{B}(\{\vec{p}\})=\sum_{\{\vec{f}\}} \mathrm{d} \sigma_{B}(\{\vec{a}\}), \quad \text { where } \quad \mathrm{d} \sigma_{B}(\{\vec{a}\})=\mathrm{d} \Phi_{B}(\{\vec{p}\}) \mathrm{B}(\{\vec{a}\}),
$$

The individual terms in the sum are given by

$$
\begin{aligned}
\mathrm{B}(\{\vec{a}\}) & =\mathcal{L}(\{\vec{a}\}) \mathcal{B}(\{\vec{a}\}), \\
\mathcal{B}(\{\vec{a}\}) & =\frac{1}{F(\{\vec{p}\})} \frac{1}{S(\{\vec{f}\})}\left|\mathcal{M}_{B}\right|^{2}(\{\vec{a}\}), \\
\mathrm{d} \Phi_{B}(\{\vec{p}\}) & =\frac{\mathrm{d} x_{1}}{x_{1}} \frac{\mathrm{d} x_{2}}{x_{2}} \mathrm{~d} \Phi_{B}(\{\vec{p}\}), \\
\mathcal{L}\left(\{\vec{a}\} ; \mu^{2}\right) & =x_{1} f_{f_{1}}\left(x_{1}, \mu^{2}\right) x_{2} f_{f_{2}}\left(x_{2}, \mu^{2}\right),
\end{aligned}
$$

where $\left|\mathcal{M}_{B}\right|^{2}(\{\vec{a}\})$ denotes the partonic matrix element squared, $\mathrm{d} \Phi_{B}(\{\vec{p}\})$ is the corresponding differential $n$-particle partonic phase-space element, $S(\{\vec{f}\})$ is the symmetry factor, $F(\{\vec{p}\})$ is the flux factor, and $\mathcal{L}$ is the parton luminosity. ${ }^{1}$

In a similar fashion, the real-emission part of the QCD next-to-leading order cross section can be written as a sum, depending on parton configurations $\left\{A_{1}, \ldots, A_{n+1}\right\}$, by replacing the Born-level matrix elements $\mathcal{B}$ with the real-emission matrix elements $\mathcal{R}$ and the Born-level phase space $\mathrm{d} \Phi_{B}$ with the real-emission phase-space $\mathrm{d} \Phi_{R}$.

It is then useful to introduce a notation for mappings from real-emission parton configurations to Born-level parton configurations and vice versa. They are given by (cf. [40])

$$
b_{i j, k}(\{\vec{A}\})=\left\{\begin{array}{c}
\{\vec{F}\} \backslash\left\{F_{i}, F_{j}\right\} \cup\left\{f_{\widetilde{\imath \jmath}}\right\} \\
\{\vec{P}\} \rightarrow\{\vec{p}\}
\end{array} \text { and } r_{\widetilde{\imath}, \tilde{k}}\left(F_{i}, \Phi_{R \mid B}^{i j, k} ;\{\vec{a}\}\right)=\left\{\begin{array}{c}
\{\vec{f}\} \backslash\left\{f_{\widetilde{\jmath}}\right\} \cup\left\{F_{i}, F_{j}\right\} \\
\{\vec{p}\} \rightarrow\{\vec{P}\}
\end{array}\right.\right.
$$

\footnotetext{
${ }^{1}$ In the case of leptonic initial states, and ignoring QED initial-state radiation, the parton distribution functions $f\left(x, \mu^{2}\right)$ are replaced by $\delta(1-x)$.
} 
The map $b_{i j, k}(\{\vec{A}\})$ combines the partons $A_{i}$ and $A_{j}$ into a common "mother" parton $a_{\widetilde{\imath \jmath}}$, in the presence of the spectator $A_{k}$ by defining a new flavour $f_{\widetilde{\imath \jmath}}$ and by redefining the particle momenta. The inverse map, $r_{\widetilde{\imath}, \tilde{k}}(\{\vec{a}\})$ determines the parton configuration of a real-emission subprocess from a Born parton configuration and a related branching process $\widetilde{\imath \jmath}, \tilde{k} \rightarrow i j, k$. The radiative variables $\Phi_{R \mid B}^{i j, k}$, denoted $\Phi_{1}$ in section 2 for brevity, are thereby employed to turn the $n$-parton momentum configuration into an $(n+1)$-parton momentum configuration.

The real-emission matrix elements, $\mathcal{R}(\{\vec{A}\})$, can be approximated in the soft and collinear limits by subtraction terms $\mathcal{D}_{i j, k}^{(\mathrm{S})}(\{\vec{A}\})$, which capture the universal singularity structure when two partons $i$ and $j$ become collinear, or one of them becomes soft in the presence of a spectator parton $k$.

$$
\mathcal{R} \stackrel{i j \text { collinear }}{i, j \text { soft }} \longrightarrow \mathcal{D}_{i j, k}^{(\mathrm{S})}(\{\vec{A}\}) .
$$

These subtraction terms are related to the ones defined in section 2 through $\mathrm{D}^{(\mathrm{S})} \rightarrow$ $\mathcal{L}(\{\vec{A}\}) \mathcal{D}_{i j, k}^{(\mathrm{S})}(\{\vec{A}\})$, with implicit notation of dipole indices, phase space and flavour configuration on the left hand side. They are not uniquely defined, but can be constructed, for example, using the Catani-Seymour method [41, 42] or the FKS approach [43, 44]. Furthermore they can be restricted in phase space [58] or extended with an arbitrary finite function. Similar terms can also be computed in a parton-shower approximation as

$$
\mathcal{R} \stackrel{i j \text { collinear }}{\longrightarrow} \mathcal{D}_{i j, k}^{(\mathrm{PS})}(\{\vec{A}\})=\mathcal{B}\left(b_{i j, k}(\{\vec{A}\})\right) \frac{S\left(b_{i j, k}(\{\vec{F}\})\right)}{S(\{\vec{F}\})} \frac{1}{2 p_{i} p_{j}} 8 \pi \alpha_{s} \mathcal{K}_{i j, k}\left(p_{i}, p_{j}, p_{k}\right),
$$

where $\mathcal{K}_{i j, k}$ denote the parton-shower evolution kernels. The parton-shower approximation is meaningful only in the collinear region, as it implements an exact factorisation of the colour structure into a Born part and an emission part. In many cases also a factorisation of the helicity structure is assumed.

\subsection{From fixed-order to resummation}

Section 2 only introduced expressions for the total cross section in the MC@NLO and POWHEG methods. A detailed discussion requires an analysis of the expectation value of arbitrary infra-red safe observables. The respective formulae are developed in the following.

Using the subtraction terms introduced in eq. (3.4), the expectation value of an observable $O$ is determined to next-to-leading order accuracy as

$$
\begin{aligned}
\langle O\rangle^{(\mathrm{NLO})}= & \sum_{\{\vec{f}\}} \int \mathrm{d} \Phi_{B}(\{\vec{p}\})\left[\mathrm{B}(\{\vec{a}\})+\tilde{\mathrm{V}}(\{\vec{a}\})+\sum_{\{\tilde{\imath}, \tilde{k}\}} \mathrm{I}_{\tilde{\imath}\}, \tilde{k}}^{(\mathrm{S})}(\{\vec{a}\})\right] O(\{\vec{p}\}) \\
& +\sum_{\{\vec{F}\}} \int \mathrm{d} \Phi_{R}(\{\vec{P}\})\left[\mathrm{R}(\{\vec{A}\}) O(\{\vec{P}\})-\sum_{\{i j, k\}} \mathrm{D}_{i j, k}^{(\mathrm{S})}(\{\vec{A}\}) O\left(b_{i j, k}(\{\vec{P}\})\right)\right] .
\end{aligned}
$$

where $\mathrm{I}^{(\mathrm{S})}(\{\vec{a}\})$ represent the subtraction terms $\mathrm{D}^{(\mathrm{S})}(\{\vec{A}\})$ integrated over the extraemission phase space. Note that the configurations $\{\vec{F}\},\{\vec{P}\}$ and $\{\vec{A}\}$ on the second line each include one more particle. 
This equation can be modified by adding and subtracting an additional arbitrary set of subtraction terms $\mathrm{D}^{(\mathrm{A})}(\{\vec{A}\})$ with the same kinematics mapping as $\mathrm{D}^{(\mathrm{S})}(\{\vec{A}\})$

$$
\begin{aligned}
\langle O\rangle^{(\mathrm{NLO})}= & \sum_{\{\vec{f}\}} \int \mathrm{d} \Phi_{B}(\{\vec{p}\}) \overline{\mathrm{B}}^{(\mathrm{A})}(\{\vec{a}\}) O(\{\vec{p}\}) \\
& +\sum_{\{\vec{F}\}} \int \mathrm{d} \Phi_{R}(\{\vec{P}\})\left[\mathrm{R}(\{\vec{A}\}) O(\{\vec{P}\})-\sum_{\{i j, k\}} \mathrm{D}_{i j, k}^{(\mathrm{A})}(\{\vec{A}\}) O\left(b_{i j, k}(\{\vec{P}\})\right)\right],
\end{aligned}
$$

where the function $\overline{\mathrm{B}}^{(\mathrm{A})}(\{\vec{a}\})$ is defined as

$$
\begin{aligned}
\overline{\mathrm{B}}^{(\mathrm{A})}(\{\vec{a}\})= & \mathrm{B}(\{\vec{a}\})+\tilde{\mathrm{V}}(\{\vec{a}\})+\sum_{\{\widetilde{\imath}, \tilde{k}\}} \mathrm{I}_{\widetilde{\imath}, \tilde{k}}^{(\mathrm{S})}(\{\vec{a}\}) \\
& +\sum_{\{\widetilde{\imath}, \tilde{k}\}} \sum_{f_{i}=q, g} \int \mathrm{d} \Phi_{R \mid B}^{i j, k}\left[\mathrm{D}_{i j, k}^{(\mathrm{A})}\left(r_{\widetilde{\imath \jmath}, \tilde{k}}(\{\vec{a}\})\right)-\mathrm{D}_{i j, k}^{(\mathrm{S})}\left(r_{\widetilde{\imath \jmath}, \tilde{k}}(\{\vec{a}\})\right)\right],
\end{aligned}
$$

and where $\mathrm{d} \Phi_{R \mid B}^{i j, k}$ represents an integral over the radiative phase space.

When combining fixed-order calculations with resummation, the task is to define a unique starting condition for the parton shower. As was argued in [11], it is not possible to process the terms $\mathrm{R}(\{\vec{A}\})$ and $\mathrm{D}_{i j, k}^{(\mathrm{A})}(\{\vec{A}\})$ in eq. (3.7) separately because this would lead to double-counting. Instead, the problem is solved if the observables on the second line of eq. (3.7) are both assumed to depend on the momentum configuration $\{\vec{P}\}$. This leads to

$$
\begin{aligned}
\langle O\rangle^{(\mathrm{NLO})}= & \sum_{\{\vec{f}\}} \int \mathrm{d} \Phi_{B}(\{\vec{p}\}) \overline{\mathrm{B}}^{(\mathrm{A})}(\{\vec{p}\}) O(\{\vec{p}\}) \\
& +\sum_{\{\vec{F}\}} \int \mathrm{d} \Phi_{R}(\{\vec{P}\})\left[\mathrm{R}(\{\vec{A}\})-\sum_{\{i j, k\}} \mathrm{D}_{i j, k}^{(\mathrm{A})}(\{\vec{A}\})\right] O(\{\vec{P}\})+\langle O\rangle^{(\text {corr })},
\end{aligned}
$$

introducing the correction term ${ }^{2}$

$$
\langle O\rangle^{(\text {corr })}=\sum_{\{\vec{F}\}} \int \mathrm{d} \Phi_{R}(\{\vec{P}\}) \sum_{\{i j, k\}} \mathrm{D}_{i j, k}^{(\mathrm{A})}(\{\vec{A}\})\left[O(\{\vec{P}\})-O\left(b_{i j, k}(\{\vec{P}\})\right)\right] .
$$

The essence of both the MC@NLO and POWHEG methods is to generate eq. (3.10) by using a Sudakov branching algorithm, which is formulated in terms of an evolution variable $t$, where $t \propto 2 p_{i} p_{j}$. Let a corresponding form factor be defined as

$$
\bar{\Delta}^{(\mathrm{A})}(t ;\{\vec{a}\})=\prod_{\{\widetilde{\imath}, \tilde{k}\}} \bar{\Delta}_{\widetilde{\imath}\}, \tilde{k}}^{(\mathrm{A})}(t ;\{\vec{a}\}),
$$

\footnotetext{
${ }^{2}$ The dependence of the observable $O$ on the kinematics of the partonic final state makes the need for a correction term $\langle O\rangle^{(\text {corr })}$ manifest. This term integrates to zero in eq. (2.9), as $O=1$ and the phase-space dependence is trivial.
} 
where

$$
\begin{aligned}
\bar{\Delta}_{\widetilde{\imath}, \tilde{k}}^{(\mathrm{A})}(t ;\{\vec{a}\})=\exp \{ & -\sum_{F_{i}=q, g} \int \mathrm{d} \Phi_{R \mid B}^{i j, k} \Theta\left(t\left(\Phi_{R \mid B}^{i j, k}\right)-t\right) \\
& \left.\times \frac{1}{S_{i j}} \frac{S\left(r_{\widetilde{\imath}, \tilde{k}}\left(F_{i} ;\{\vec{f}\}\right)\right)}{S(\{\vec{f}\})} \frac{\mathrm{D}_{i j, k}^{(\mathrm{A})}\left(r_{\widetilde{\imath}, \tilde{k}}\left(F_{i}, \Phi_{R \mid B}^{i j, k} ;\{\vec{a}\}\right)\right)}{\mathrm{B}(\{\vec{a}\})}\right\} .
\end{aligned}
$$

The ratio of symmetry factors, including $S_{i j}$, is explained in detail in [40]. It accounts for the way in which the phase space is successively filled by a parton shower.

It can then be proven [11-13, 40], that the following formula for the expectation value of an infrared safe observable reproduces eq. (3.9) to $\mathcal{O}\left(\alpha_{s}\right)$ :

$$
\begin{aligned}
& \langle O\rangle^{(\mathrm{NLOMC})}=\sum_{\{\vec{f}\}} \int \mathrm{d} \Phi_{B}(\{\vec{p}\}) \overline{\mathrm{B}}^{(\mathrm{A})}(\{\vec{a}\})[\underbrace{\bar{\Delta}^{(\mathrm{A})}\left(t_{0} ;\{\vec{a}\}\right)}_{\text {unresolved }} O(\{\vec{p}\}) \\
& +\sum_{\{\widetilde{\imath}, \tilde{k}\}} \sum_{F_{i}=q, g} \int \mathrm{d} \Phi_{R \mid B}^{i j, k} \Theta\left(t\left(\Phi_{R \mid B}^{i j, k}\right)-t_{0}\right) O\left(r_{\widetilde{\imath \jmath}, \tilde{k}}(\{\vec{p}\})\right) \\
& \times \underbrace{\frac{1}{S_{i j}} \frac{S\left(r_{\widetilde{\imath}, \tilde{k}}\left(F_{i} ;\{\vec{f}\}\right)\right)}{S(\{\vec{f}\})} \frac{\mathrm{D}_{i j, k}^{(\mathrm{A})}\left(r_{\widetilde{\imath}, \tilde{k}}\left(F_{i}, \Phi_{R \mid B}^{i j, k} ;\{\vec{a}\}\right)\right)}{\mathrm{B}(\{\vec{a}\})} \bar{\Delta}^{(\mathrm{A})}(t ;\{\vec{a}\})}_{\text {resolved, singular }}] \\
& +\sum_{\{\vec{F}\}} \int \mathrm{d} \Phi_{R}(\{\vec{P}\}) \underbrace{\left[\mathrm{R}(\{\vec{A}\})-\sum_{i j, k} \mathrm{D}_{i j, k}^{(\mathrm{A})}(\{\vec{A}\})\right]}_{\text {resolved, non-singular }} O(\{\vec{P}\}) .
\end{aligned}
$$

NLO Monte-Carlo events reproducing eq. (3.13) can be generated in the following way:

- A seed event is produced according to either the first or the second line of eq. (3.9), not including the correction factor $\langle O\rangle^{(\text {corr })}$.

- If the second line is chosen (HI-event) the event has real-emission kinematics and is kept as-is.

This generates the "resolved, non-singular" term of eq. (3.13)

- If the first line is chosen ( $\mathbb{S}$-event), the event has Born-like kinematics and is processed through a one-step Sudakov branching algorithm described by eqs. (3.11) and (3.12). The necessary techniques for that case will be detailed in section 3.4. An emission might or might not occur, which is represented by the "resolved, singular" and "unresolved" terms of eq. (3.13), respectively.

\subsection{The MC@NLO and PowHEG methods}

Equation (3.13) becomes particularly simple when

$$
\mathrm{D}_{i j, k}^{(\mathrm{A})} \rightarrow \mathrm{D}_{i j, k}^{(\mathrm{S})}
$$


In this special case the integral over the radiative phase space in eq. (3.8) is zero. We will refer to this as the MC@NLO technique. It can be thought of as a method which uses the Sudakov branching probability for subtraction or, conversely, which uses the subtraction kernels for parton evolution. Possible event generation techniques to achieve this will be discussed in section 3.4.

The POwHEG method, in its original form, on the other hand, defines

$$
\mathrm{D}_{i j, k}^{(\mathrm{A})} \rightarrow \rho_{i j, k}(\{\vec{A}\}) \mathrm{R}(\{\vec{A}\}) \quad \text { where } \quad \rho_{i j, k}(\{\vec{A}\})=\frac{\mathcal{D}_{i j, k}^{(\mathrm{S})}(\{\vec{A}\})}{\sum_{m n, l} \mathcal{D}_{m n, l}^{(\mathrm{S})}(\{\vec{A}\})},
$$

It can be thought of as exponentiating the full radiative corrections into a Sudakov form factor. As such, it bears strong similarity to matrix-element corrected power showers. This aspect has been discussed extensively in [40, 59].

One can construct a mixed scheme, where $\mathrm{D}_{i j, k}^{(\mathrm{A})}$ is defined as

$$
\mathrm{D}_{i j, k}^{(\mathrm{A})} \rightarrow \rho_{i j, k}(\{\vec{A}\})\left[\mathrm{R}(\{\vec{A}\})-\mathrm{R}^{(r)}(\{\vec{A}\})\right],
$$

with $\mathrm{R}^{(r)}$ an arbitrary infrared-finite part of the real-emission cross section and $\rho_{i j, k}$ given by eq. (3.15). This method leads to the original MC@NLO prescription, proposed in [11], if $\mathrm{D}_{i j, k}^{(\mathrm{A})}=\mathrm{D}_{i j, k}^{(\mathrm{PS})}$. It was also used in [50] to deal with the problem of radiation zeros in the $W^{ \pm}$-production process in the POWHEG approach.

Note that all the above choices of $\mathrm{D}_{i j, k}^{(\mathrm{A})}$ fulfil the requirement that the kinematics and flavour mapping be identical to the one in $\mathrm{D}_{i j, k}^{(\mathrm{S})}$, cf. eq. (3.7).

\subsection{Event-generation techniques}

In the parton-shower approximation, eq. (3.12) would read

$$
\begin{aligned}
\Delta_{\widetilde{\imath}, \tilde{k}}\left(t^{\prime}, t^{\prime \prime} ;\{\vec{a}\}\right)=\exp \{ & -\sum_{F_{i}=q, g} \int_{t^{\prime}}^{t^{\prime \prime}} \frac{\mathrm{d} t}{t} \int_{z_{\min }}^{z_{\max }} \mathrm{d} z \int_{0}^{2 \pi} \frac{\mathrm{d} \phi}{2 \pi} J_{i j, k}(t, z, \phi) \\
& \left.\times \frac{1}{S_{i j}} \frac{\alpha_{s}}{2 \pi} \mathcal{K}_{i j, k}(t, z, \phi) \frac{\mathcal{L}\left(r_{\widetilde{\imath}, \tilde{k}}\left(F_{i}, t, z, \phi ;\{\vec{a}\}\right) ; t\right)}{\mathcal{L}(\{\vec{a}\} ; t)}\right\},
\end{aligned}
$$

where $z$ is called the splitting variable of the parton-shower model and $J_{i j, k}$ is the Jacobian factor associated with the transformation of $\mathrm{d} \Phi_{R \mid B}^{i j, k}$ into $\mathrm{d} t \mathrm{~d} z \mathrm{~d} \phi$ [40].

It is well known how to generate emissions according to eq. (3.17). The task of a proper implementation of MC@NLO and POWHEG is, however, to employ eq. (3.12). In our formulation of $\mathrm{MC} @ \mathrm{NLO}$, i.e. with $\mathrm{D}_{i j, k}^{(\mathrm{A})}=\mathrm{D}_{i j, k}^{(\mathrm{S})}$, this can involve the exponentiation of subtraction terms which are negative, due to subleading colour configurations in the real-emission matrix elements, cf. section 2.5. This leads to a form factor larger than one, which cannot be interpreted in terms of a no-branching probability. In this section we will show how to generate such form factors efficiently in a Markov chain Monte Carlo. The respective algorithm is the basis for our implementation of the MC@NLO method. It was outlined in [60] and reformulated in [61]. As it needs to be modified slightly in the current context, it is briefly discussed here again. The method is based on an extension of the veto algorithm [62]. 
The veto algorithm. Let $t$ be the parton-shower evolution variable and $f(t)$ the splitting kernel $\mathcal{K}$, integrated over the splitting variable $z .^{3}$ The differential probability for generating a branching at scale $t$, when starting from an upper evolution scale $t^{\prime}$ is then given by

$$
\mathcal{P}\left(t, t^{\prime}\right)=f(t) \exp \left\{-\int_{t}^{t^{\prime}} \mathrm{d} \bar{t} f(\bar{t})\right\} .
$$

A new scale $t$ is therefore found as

$$
t=F^{-1}\left[F\left(t^{\prime}\right)+\log \#\right] \quad \text { where } \quad F(t)=\int_{t} \mathrm{~d} \bar{t} f(\bar{t}),
$$

and where \# is a random number between zero and one. The key point of the veto algorithm is, that even if the integral $F(t)$ is unknown, one can still generate events according to $\mathcal{P}$ using an overestimate $g(t) \geq f(t)$ with a known integral $G(t)$. Firstly, a value $t$ is generated as $t=G^{-1}\left[G\left(t^{\prime}\right)+\log \#\right]$. Secondly, the value is accepted with probability $f(t) / g(t)$. A splitting at $t$ with $n$ intermediate rejections is then produced with probability

$$
\begin{aligned}
\mathcal{P}_{n}\left(t, t^{\prime}\right)= & \frac{f(t)}{g(t)} g(t) \exp \left\{-\int_{t}^{t_{1}} \mathrm{~d} \bar{t} g(\bar{t})\right\} \\
& \times \prod_{i=1}^{n}\left[\int_{t_{i-1}}^{t_{n+1}} \mathrm{~d} t_{i}\left(1-\frac{f\left(t_{i}\right)}{g\left(t_{i}\right)}\right) g\left(t_{i}\right) \exp \left\{-\int_{t_{i}}^{t_{i+1}} \mathrm{~d} \bar{t} g(\bar{t})\right\}\right],
\end{aligned}
$$

where $t_{n+1}=t^{\prime}$ and $t_{0}=t$. The nested integrals in eq. (3.20) can be disentangled, and summing over $n$ leads to the exponentiation of the factor $g(t)-f(t)$, such that eq. (3.18) is reproduced [62].

Analytic weights. The purpose here is to introduce an additional overestimate $h(t)$. The additional weight $g(t) / h(t)$ is then applied analytically rather than using a hit-or-miss method. This leads to the following expression for the differential probability to generate an emission at $t$ with $n$ rejections between $t$ and $t^{\prime}$

$$
\begin{aligned}
\mathcal{P}_{n}\left(t, t^{\prime}\right)= & \frac{f(t)}{g(t)} h(t) \exp \left\{-\int_{t}^{t_{1}} \mathrm{~d} \bar{t} h(\bar{t})\right\} \\
& \times \prod_{i=1}^{n}\left[\int_{t_{i-1}}^{t_{n+1}} \mathrm{~d} t_{i}\left(1-\frac{f\left(t_{i}\right)}{g\left(t_{i}\right)}\right) h\left(t_{i}\right) \exp \left\{-\int_{t_{i}}^{t_{i+1}} \mathrm{~d} \bar{t} h(\bar{t})\right\}\right] .
\end{aligned}
$$

Note that the function $h(t)$ and the ratio $f(t) / g(t)$ must be positive definite. Generating events according to eq. (3.21) is then straightforward. In order to recover the desired distribution, the following analytic weight needs to be applied

$$
w\left(t, t_{1}, \ldots, t_{n}\right)=\frac{g(t)}{h(t)} \prod_{i=1}^{n} \frac{g\left(t_{i}\right)}{h\left(t_{i}\right)} \frac{h\left(t_{i}\right)-f\left(t_{i}\right)}{g\left(t_{i}\right)-f\left(t_{i}\right)} .
$$

\footnotetext{
${ }^{3}$ For simplicity, the existence of only one splitting function is assumed, i.e. that there is no flavour change of the splitter during the evolution. The extension to flavour changing splittings is straightforward, but it would unnecessarily complicate the notation at this point.
} 
Here the term $g(t) / h(t)$ is due to the acceptance of the emission. The product, which is needed for an exponentiation of $h(t)-f(t)$ instead of $g(t)-f(t)$, runs over all correction weights for rejected steps. Equation (3.22) can lead to negative weights, which reflect the fact that sub-leading colour configurations are taken into account and that the a-priori density $h(t)$ might underestimate $f(t)$.

Implementation of the MC@NLO method. In order to implement the MC@NLO method with $\mathrm{D}_{i j, k}^{(\mathrm{A})}=\mathrm{D}_{i j, k}^{(\mathrm{S})}$ we need to identify the function $f$ above with the $(z, \phi)$ integrated subtraction terms $\mathrm{D}_{i j, k}^{(\mathrm{S})}$, divided by the Born contribution. A convenient choice of the function $h$ will be the $(z, \phi)$-integral of the parton-shower evolution kernels $\mathrm{D}_{i j, k}^{(\mathrm{PS})}$, divided by the Born contribution. We are then free to choose the auxiliary function $g$ on a point-by-point basis, but a convenient way is to define $g=C f$, where $C$ is a constant larger than one. This guarantees that both acceptance and rejection term are generated in sufficient abundance to reduce Monte-Carlo errors. In the remainder of this paper, the functions $f, g$ and $h$ are thus schematically identified as follows:

$$
f \rightarrow \mathrm{D}^{(\mathrm{A})}, \quad h \rightarrow \mathrm{D}^{(\mathrm{PS})}, \quad \text { and } \quad g=C f,
$$

where $C \approx 2$ is a constant.

By exponentiating $\mathrm{D}_{i j, k}^{(\mathrm{S})}$ in this manner, we can suppress the integral over the radiative phase space in eq. (3.8) and thus substantially simplify the event generation.

\section{Analysis of perturbative uncertainties}

In the following the uncertainties discussed in section 2.5 are investigated in detail. The event generator SHERPA [63, 64] sets the framework for this study, including its automated MC@NLO implementation, the matrix-element generator AMEGIC++ [65], an automated implementation [66] of the Catani-Seymour dipole subtraction method [41] and the parton shower model described in [57,60]. The CTEQ6.6 PDF set [67] is used together with the corresponding parametrisation of the running coupling. All analyses are carried out with the help of Rivet [68].

\subsection{Higgs-boson production in gluon fusion}

The production of a Higgs boson at the LHC with $7 \mathrm{TeV}$ centre-of-mass energy serves as a first example. This process amplifies some of the effects discussed in section 2.5 because the radiating partons are gluons. There are thus no valence PDF's involved at Born level, which allows to test fixed-order effects and resummation in a relatively clean setting. Results presented in this section do not include non-perturbative or multiple parton scattering effects. A detailed study of the POWHEG algorithm and its associated uncertainties has already been presented in [40]. In the following, the focus will therefore mainly lie on our implementation of the MC@NLO method, but comparisons with POWHEG will be made at the appropriate junctures.

For an unbiased comparison of the MC@NLO and POWHEG techniques, the events are analysed with a minimal set of cuts. A Higgs-boson mass of $m_{h}=120 \mathrm{GeV}$ is assumed and 
two $\tau$-leptons with $|\eta|<3.5$ and $p_{\perp}>25 \mathrm{GeV}$ are required; jets are defined according to the inclusive $k_{\perp}$-algorithm [69-71] with $R=0.7$ and $p_{\perp \text {,min }}=20 \mathrm{GeV}$. The renormalisation and factorisation scales are set to $\mu_{F}=\mu_{R}=m_{h}$. The effective coupling of the Higgs to gluons, mediated by a top-quark loop, is modeled through an effective Lagrangian [72, 73]. Both powers of $\alpha_{s}$ in the effective gluon-gluon-Higgs coupling are also evaluated at $m_{h}$.

The comparison with fixed-order predictions and predictions from standard parton showers (referred to as $\mathrm{LO} \otimes \mathrm{PS}$ in the following) presents a first crucial test of the MC@NLO and Powheg methods. In this context, SHERPA is also used as a framework for NLO fixed-order event generation, enabling a comparison of all approaches with identical input parameters. In order to vary the amount of exponentiated real-emission corrections in our MC@NLO, which is governed by eqs. (2.10)-(2.12) or, more rigorous, eqs. (3.12)-(3.14), the phase-space restriction described in [58] is employed and the parameter $\alpha_{\text {cut }}$ is varied. It has the effect of increasing $\left(\alpha_{\text {cut }} \rightarrow 1\right)$ or decreasing $\left(\alpha_{\text {cut }} \rightarrow 0\right)$ the phase space for nonsingular contributions in $\mathrm{D}^{(\mathrm{A})}$ by setting an upper bound on the virtuality $\hat{t}$ of the splitting parton. The leading logarithm is then of the form $\alpha_{s} \log ^{2}\left(\hat{t} / \alpha_{\text {cut }} s\right)$, with $s$ the centre-ofmass energy of the colliding protons. Hence, for $\alpha_{\text {cut }} \sim 1$ the leading logarithm contains the wrong argument $\alpha_{\text {cut }} s \gg \mu_{F}^{2}$, clearly at odds with usual choices of the resummation scale and in violation of factorisation theorems. Similarly, the original POWHEG method exponentiates radiative corrections throughout the real-emission phase space, corresponding to our Mc@Nlo with $\alpha_{\text {cut }}=1$. Some practical implementations of POWHEG propose to suppress non-singular terms with a continuous function which tends to one in the singular limits and approaches zero in those regions of phase space where emissions become hard [50]. However, even in this approach the volume of phase space where exponentiation is performed is left invariant, and only the influence of non-logarithmic terms is reduced. The leading logarithm is thus still of the form $\alpha_{s} \log ^{2}(\hat{t} / s)$. The traditional LO $\otimes \mathrm{PS}$ method directly implements the DGLAP resummation and thus uses the factorisation scale $\mu_{F}$ as a phase space constraint, resulting in the correct logarithmic form $\alpha_{s} \log ^{2}\left(\hat{t} / \mu_{F}^{2}\right)$. It is clearly desirable to implement this very same constraint in our MC@NLO. This requires the computation of a new class of integrated subtraction terms in the Catani-Seymour framework, which is beyond the scope of this work and will be the topic of a forthcoming publication.

The influence of $\alpha_{\text {cut }}$ on the MC@NLO predictions and its relation to the PowHEG and $\mathrm{LO} \otimes \mathrm{PS}$ predictions are investigated for various observables in figures $1-5$. Taking the $\mathcal{O}\left(\alpha_{s}\right)$ expansion of eq. (3.13) at face value leads to the expectation that results from MC@NLO (for any $\alpha_{\text {cut }}$ ) and PoWHEG should approximately agree with fixed-order predictions in the limit of hard, well separated partons. In the limit of soft/collinear radiation the common logarithmic structure of the resummation in MC@NLO, POWHEG and LO $\otimes$ PS should lead to identical results in all three approaches.

In practice, the transverse momentum spectrum of the Higgs boson, shown in figure 1a, exhibits a large dependence on $\alpha_{\text {cut }}$. This roots in the different composition of the distribution in terms of $\mathbb{H}$ - and $\mathbb{S}$-events, which is depicted in figures 6 and 7 . Neglecting subsequent parton shower emissions, $\mathbb{H}$-events above $\alpha_{\text {cut }}$ coincide with those of the NLO calculation, while $\mathbb{S}$-events resum the leading logarithm of the real emission matrix element and occur with an additional enhancement $\overline{\mathrm{B}}^{(\mathrm{A})} / \mathrm{B}$. This enhancement, though formally 


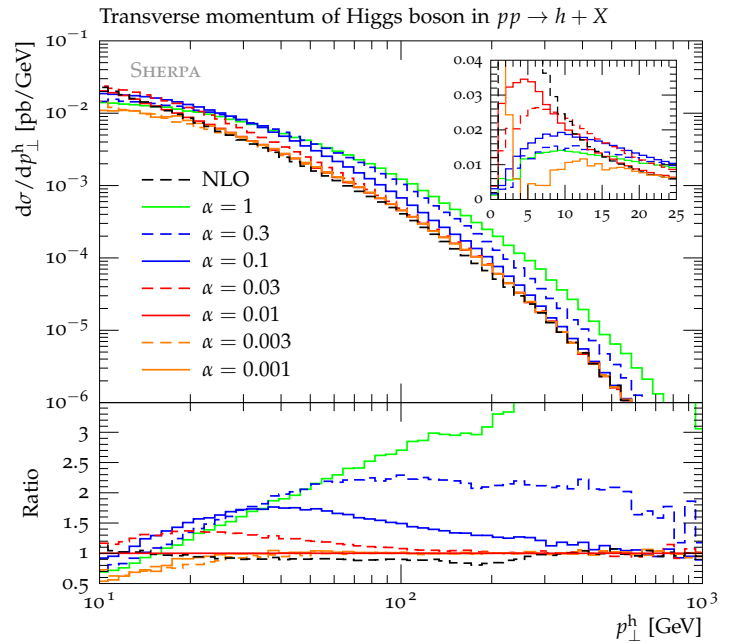

(a)

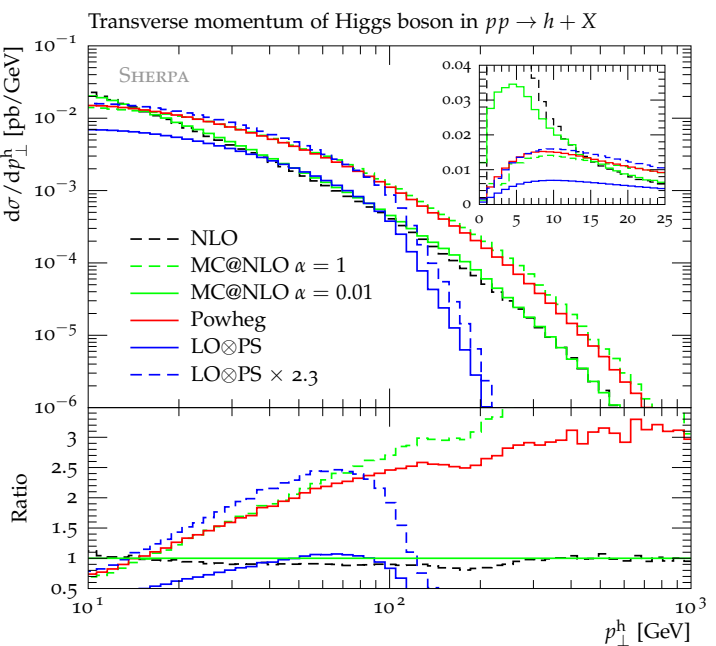

(b)

Figure 1. Transverse momentum of the Higgs boson in inclusive Higgs boson production $\left(m_{h}=\right.$ $120 \mathrm{GeV}$ ) at $E_{\mathrm{cms}}=7 \mathrm{TeV}$. The variation of $\mathrm{MC} @ \mathrm{NLO}$ predictions with varying $\alpha_{\mathrm{cut}}$ (denoted $\alpha$ in the legend) is shown in figure (a), while figure (b) compares the MC@NLO, POwHEG and $\mathrm{LO} \otimes \mathrm{PS}$ methods.

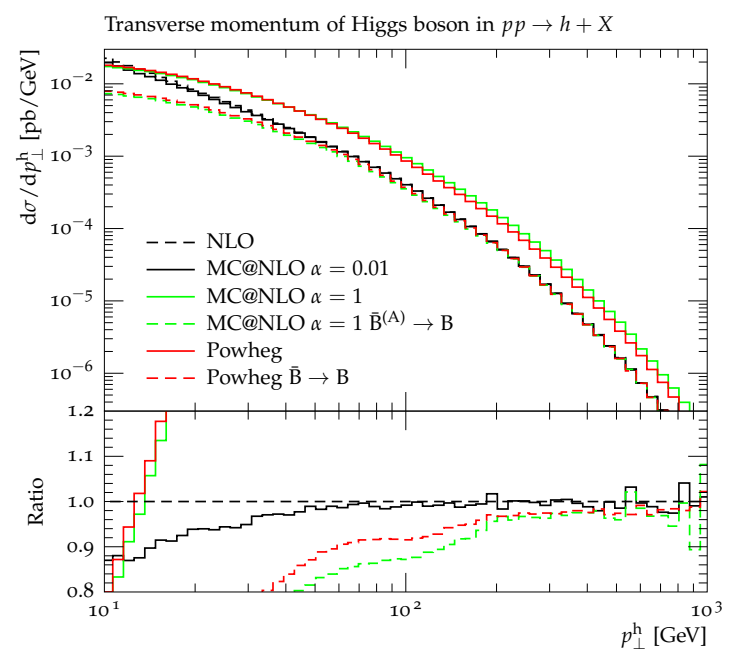

Figure 2. Transverse momentum of the Higgs boson (left) in inclusive Higgs boson production $\left(m_{h}=120 \mathrm{GeV}\right)$ at $E_{\mathrm{cms}}=7 \mathrm{TeV}$. The solid lines show the full MC@NLO (green) and PowHEG (red) results, while the corresponding dashed lines show the results where the prefactor of the resummed $\mathbb{S}$ events, $\overline{\mathrm{B}}$ and $\overline{\mathrm{B}}^{(\mathrm{A})}$ respectively, have been replaced by B. In all cases only the first emission is taken into account. The black dashed line shows the NLO fixed-order prediction.

contributing to $\mathcal{O}\left(\alpha_{s}^{2}\right)$, can be numerically large. Thus, when allowing $\mathbb{S}$-type events to fill more than the phase space suited for the resummation of the DGLAP logarithms, their emission probability is not only distorted by the exponentiation but also by an artificial 


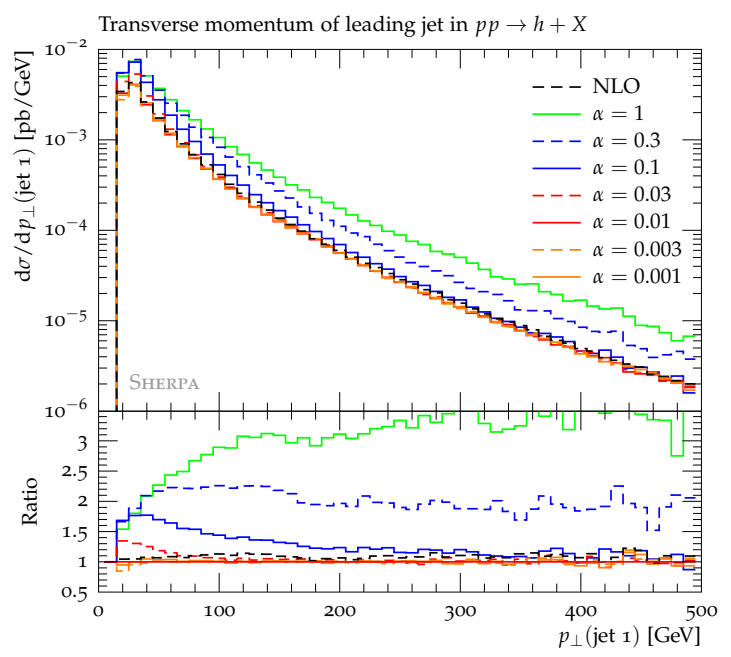

(a)

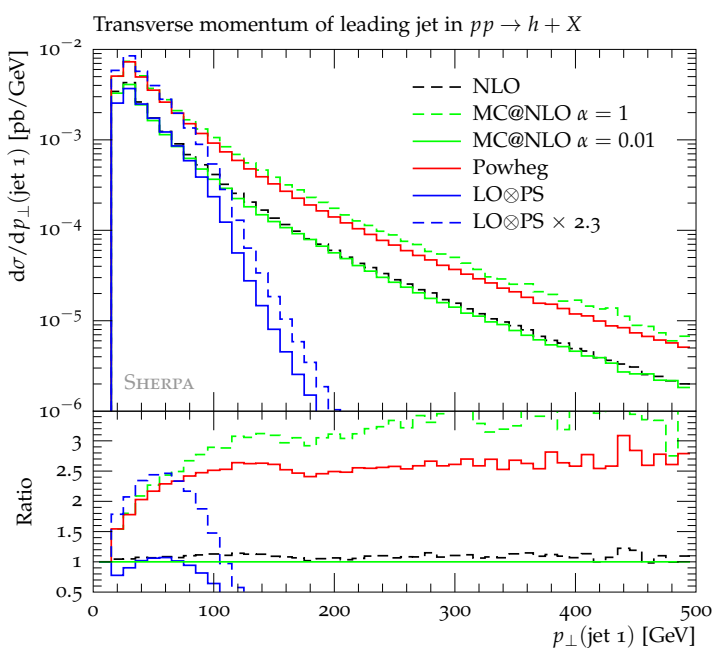

(b)

Figure 3. Transverse momentum of the leading jet in inclusive Higgs-boson production $\left(m_{h}=\right.$ $120 \mathrm{GeV}$ ) at $E_{\mathrm{cms}}=7 \mathrm{TeV}$. See figure 1 for details.

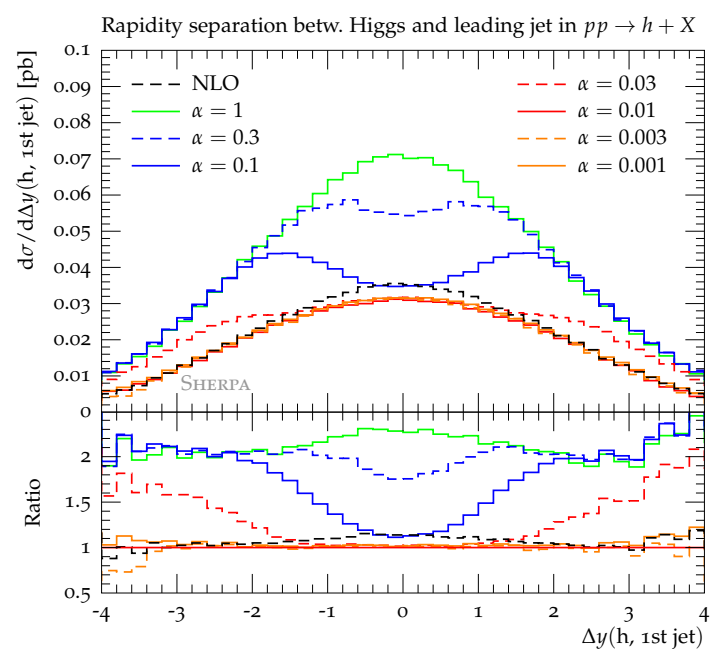

(a)

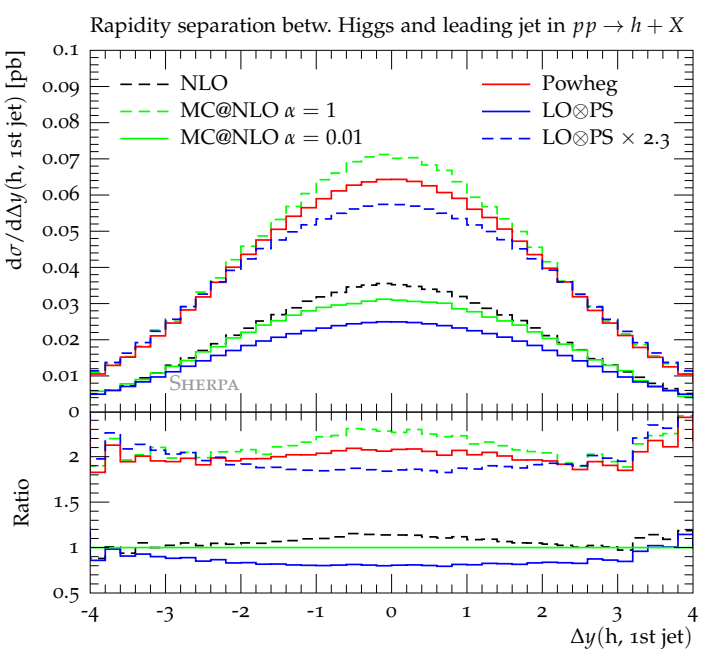

(b)

Figure 4. Rapidity separation between Higgs-boson and leading jet in inclusive Higgs-boson production $\left(m_{h}=120 \mathrm{GeV}\right)$ at $E_{\mathrm{cms}}=7 \mathrm{TeV}$. See figure 1 for details.

$K$-factor $\overline{\mathrm{B}}^{(\mathrm{A})} / \mathrm{B}$, as compared to the NLO result. For smaller values of $\alpha_{\text {cut }}$, the argument of the leading logarithm is closer to the one in DGLAP evolution, even though $\alpha_{\text {cut }}$ has a functional form which makes a direct comparison difficult. It is thus observed that the MC@NLO distributions come close to the fixed-order result in the hard region, and the method behaves exactly as expected.

Further, excessively small values of $\alpha_{\text {cut }}$ lead to spurious results, as the $\mathbb{S}$-event prefactor $\overline{\mathrm{B}}^{(\mathrm{A})} / \mathrm{B}$ turns negative. This is exemplified in figure 6 . Thus, those values of $\alpha_{\text {cut }}$ are pre- 


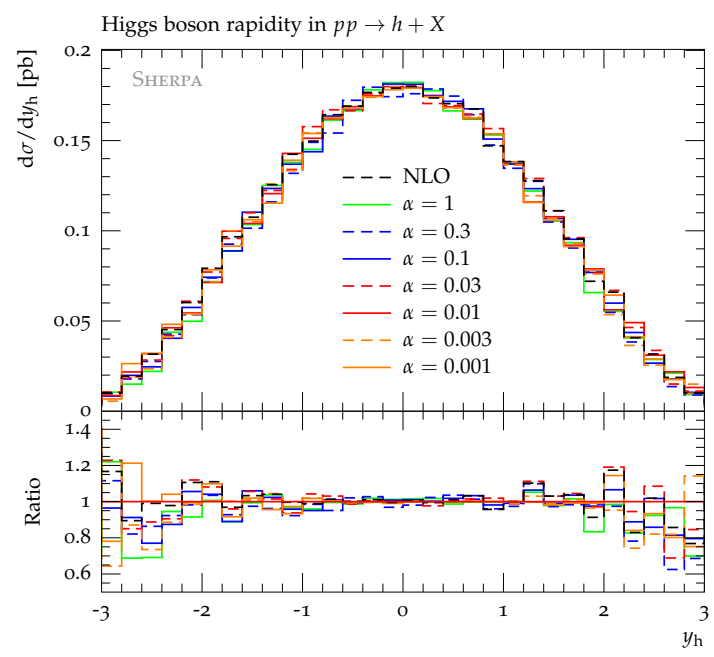

(a)

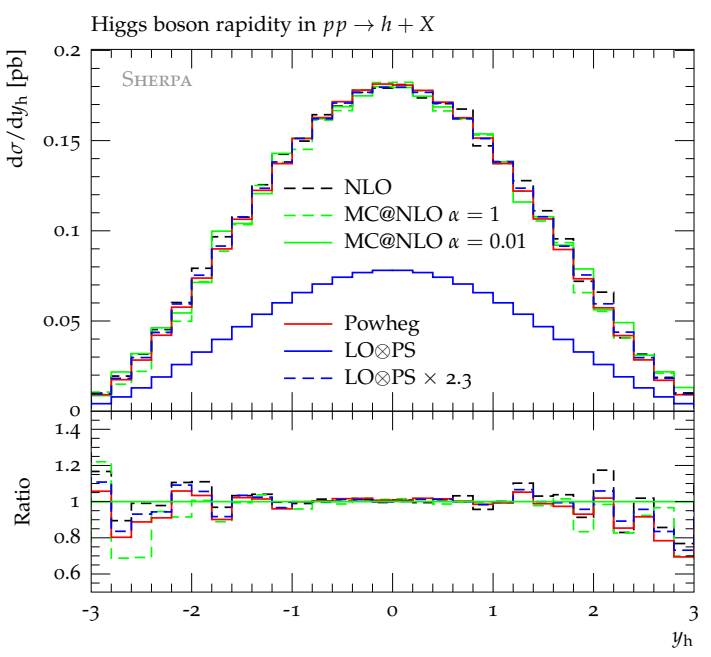

(b)

Figure 5. Prediction and uncertainties for the rapidity of the Higgs boson in inclusive Higgs boson production $\left(m_{h}=120 \mathrm{GeV}\right)$ at $E_{\mathrm{cms}}=7 \mathrm{TeV}$. See figure 1 for details.

ferred which ensure that $\mathbb{S}$-events only fill the phase space suited for resummation and simultaneously avoid $\overline{\mathrm{B}}^{(\mathrm{A})}$ turning negative. Defining such an allowed range of $\alpha_{\text {cut }}$ introduces an unwanted process dependence into this implementation. Clearly a physically more meaningful definition of phase space constraints will ultimately lead to removing this uncertainty.

Comparing these results to the predictions of the POWHEG and LO $\otimes \mathrm{PS}$ methods, depicted in figure 1b, one finds that POWHEG behaves as our MC@NLO implementation with $\alpha_{\text {cut }}=1$, suffering from a too large phase space for exponentiation. On the other hand, the resummation properties of the parton shower are retained. The difference in the actual Sudakov shape can entirely be attributed to the respective size and functional form of the phase-space boundaries. Further, within the region where the standard parton shower is able to fill the phase space (up to $|\hat{t}|=\mu_{F}^{2}$ ), the $\mathrm{LO} \otimes \mathrm{PS}$ result scaled by a global factor $K=\sigma_{\mathrm{NLO}} / \sigma_{\mathrm{LO}}=2.3$ is in good agreement with the POWHEG and MC@NLO with $\alpha_{\text {cut }}=1$ distributions. This is a consequence of the simple form of the virtual corrections resulting in a local $K$-factor $\overline{\mathrm{B}}^{(\mathrm{A})} / \mathrm{B}$, that largely coincides with the global $K$-factor.

It was claimed in [56] that the difference between POWHEG and MC@NLO predictions for Higgs-boson production originates from the local $K$-factor $\overline{\mathrm{B}} / \mathrm{B}$ in POWHEG. Figure 2 shows explicitly that this claim is at odds with our findings. It displays predictions from a standard POwHEg simulation and a modified one, where we replace $\overline{\mathrm{B}} \rightarrow \mathrm{B}$. The same analysis is repeated with MC@NLO at $\alpha=1$, except that in this case we replace $\overline{\mathrm{B}}^{(\mathrm{A})} \rightarrow \mathrm{B}$. It is manifest that the original and the modified POWHEG results agree, except for a global $K$-factor, as was observed in [56]. However, the same effect is found for MC@NLO at $\alpha=1$. In contrast, both the modified POWHEG and the modified MC@NLO result differ in shape from the NLO prediction at high $p_{T}$, while the MC@NLO result with $\alpha=0.01$ matches the NLO result exactly. The deviations between POWHEG and MC@NLO, which were observed in [56] can therefore not be attributed to the local $K$-factor $\overline{\mathrm{B}} \rightarrow \mathrm{B}$. Instead they must originate from the unrestricted phase space in POWHEG. 

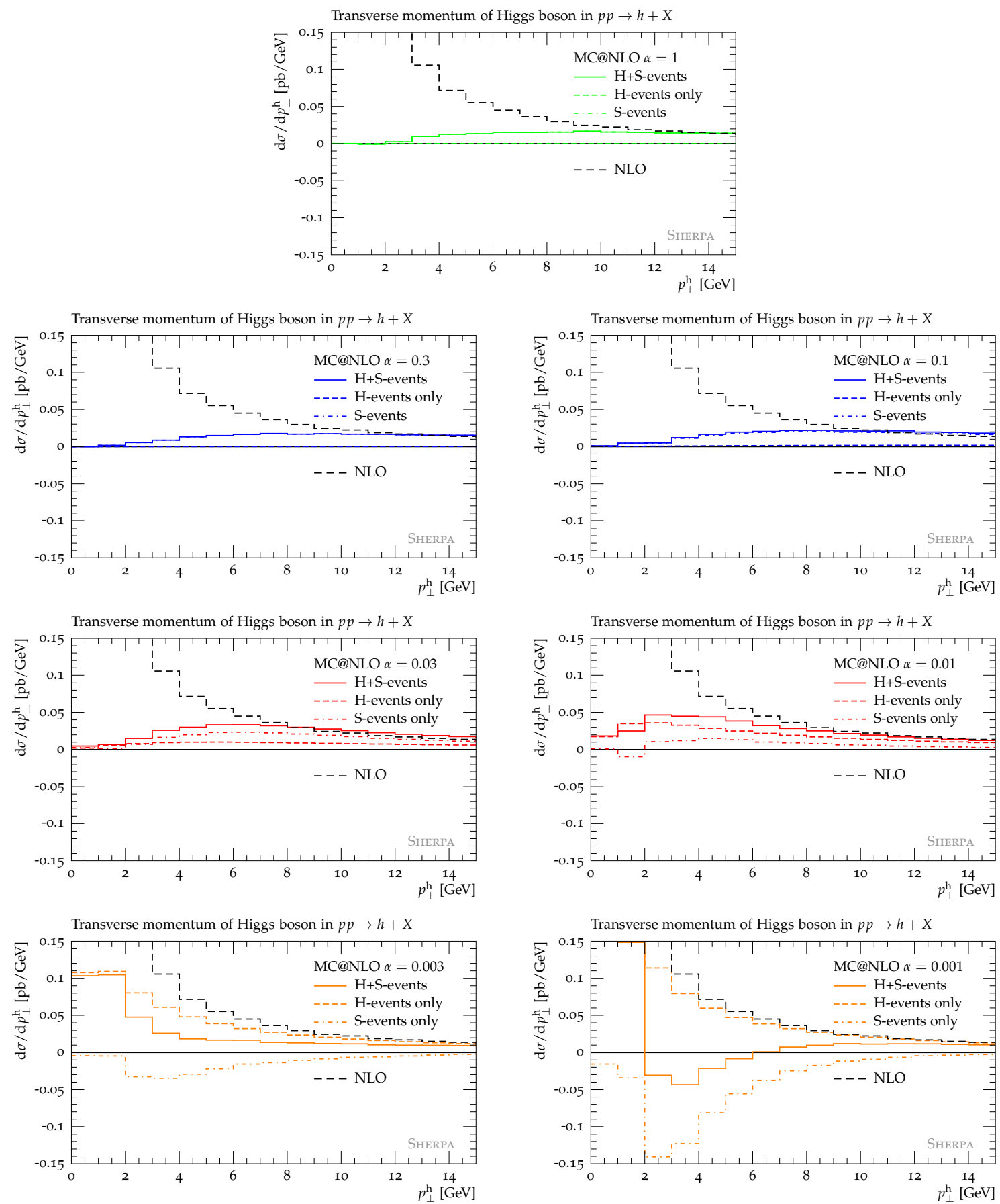

Figure 6. Higgs-boson transverse momentum for small values of $p_{\perp}^{\mathrm{h}}$ in inclusive Higgs-boson production $\left(m_{h}=120 \mathrm{GeV}\right)$ at $E_{\mathrm{cms}}=7 \mathrm{TeV}$. The contribution of $\mathbb{S}$ - and $\mathbb{H}$-events in the MC@NLO method is displayed separately for different values of $\alpha_{\text {cut }}$. For $\alpha_{\text {cut }}=1$ and $\alpha_{\text {cut }}=0.3$ the sample consist primarily of $\mathbb{S}$-events with small additions of negatively and positively valued $\mathbb{H}$-events, respectively. 

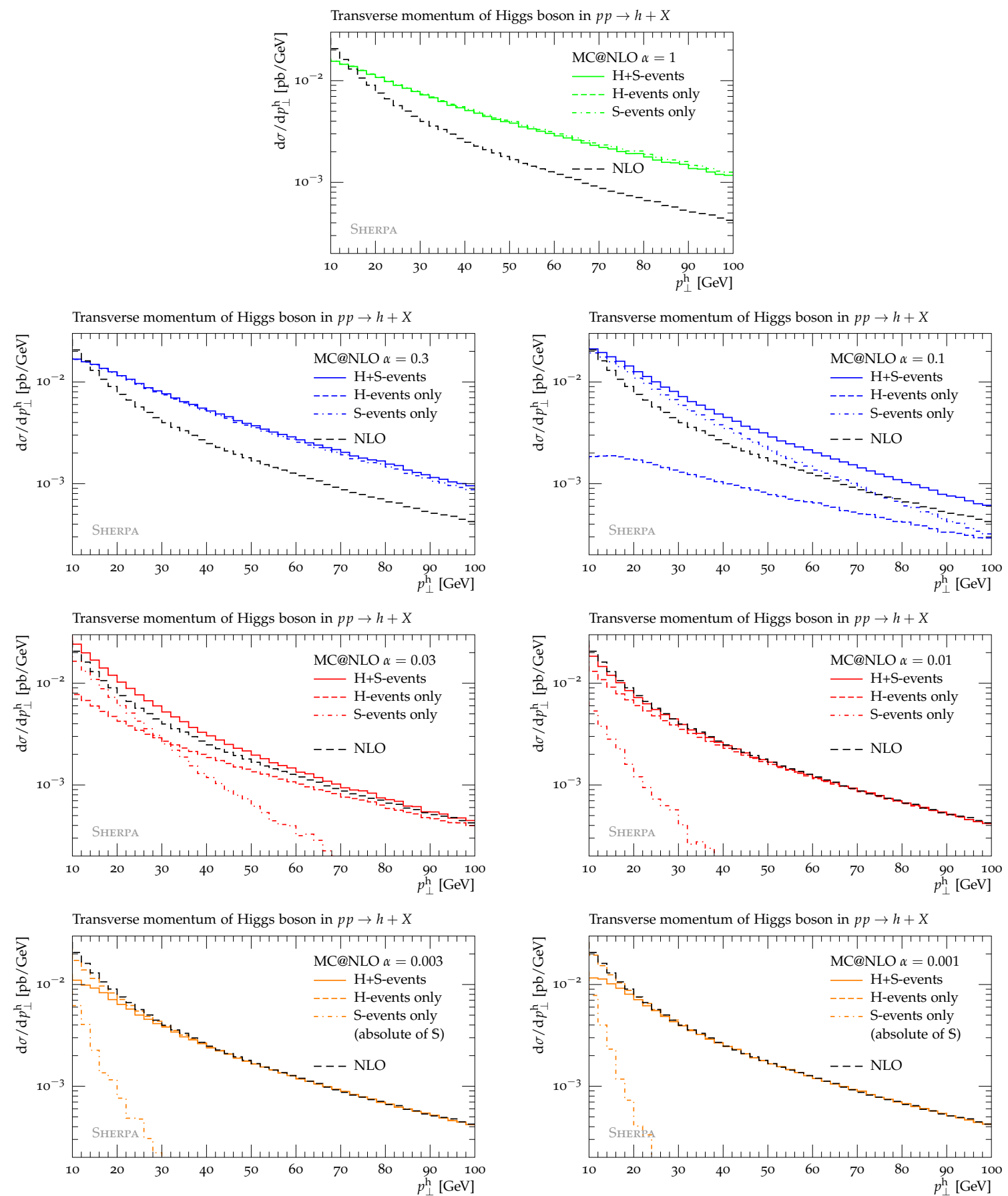

Figure 7. Higgs-boson transverse momentum for large values of $p_{\perp}^{\mathrm{h}}$ in inclusive Higgs-boson production $\left(m_{h}=120 \mathrm{GeV}\right)$ at $E_{\mathrm{cms}}=7 \mathrm{TeV}$. The contribution of $\mathbb{S}$ - and $\mathbb{H}$-events in the MC@NLO method is displayed separately for different values of $\alpha_{\text {cut }}$. For $\alpha_{\text {cut }}=1$ and $\alpha_{\text {cut }}=0.3$ the sample consist primarily of $\mathbb{S}$-events with small additions of negatively and positively valued $\mathbb{H}$-events, respectively. 
Similar observations are made for the transverse momentum of the hardest jet of figure 3. Here, the difference in the emission rate for $\mathbb{H}$ - and $\mathbb{S}$-events, as discussed earlier, is plainly visible. Again, MC@NLO with $\alpha_{\text {cut }}=0.01$ agrees with the fixed-order NLO prediction for large jet transverse momenta. Also POWHEG and MC@NLO with $\alpha_{\text {cut }}=1$ give similar results, overestimating the emission rate by more than $250 \%$.

This overestimation also feeds into the rapidity separation between the Higgs boson and the first jet in figure 4. The predictions of MC@NLO with $\alpha_{\text {cut }}=0.01$ and MC@NLO with $\alpha_{\text {cut }}=1$ differ by a factor of two. The effects of decreasing $\alpha_{\text {cut }}$ are examined in figure $4 \mathrm{a}$. With decreasing $\alpha_{\text {cut }}$, the rapidity difference distribution develops a pronounced dip, which becomes broader and flattens out until the fixed-order result is approached for $\alpha_{\text {cut }} \approx 0.01$ and stabilises for even smaller $\alpha_{\text {cut }}$. Again, this is explained by the different composition of the samples in terms of $\mathbb{H}$ - and $\mathbb{S}$-events. Similar findings were reported in [74], where $t \bar{t}$-production was analysed and the MC@NLO method was compared to tree-level merging. The authors concluded that a dip in the leading jet rapidity spectrum, present only in the MC@NLO prediction, was most likely an artifact of the incomplete phasespace coverage in HERWIG's parton shower. While the dead zones of this parton shower are clearly different from the dead zones generated through $\alpha_{\text {cut }}$ in our approach, the resulting effect is surprisingly similar. It suggests that the dip, which is not present in either NLO fixed-order or tree-level merged results, must be attributed to exponentiation uncertainties. Figure $4 \mathrm{~b}$ shows a comparison with POWHEG and LO $\otimes$ PS. POWHEG predictions agree well with those from our $\mathrm{MC} @ \mathrm{NLO}$ with $\alpha_{\text {cut }}=1$ and from LO $\otimes \mathrm{PS}$ with a global $K$-factor, while MC@NLO with $\alpha_{\text {cut }}=0.01$ agrees with standard LO $\otimes$ PS.

Figure 5 shows the rapidity spectrum of the Higgs boson, which can be defined at Born level and is thus described at NLO accuracy. No significant variation is observed in this spectrum when the parameter $\alpha_{\text {cut }}$ is varied, as expected. One can thus conclude that both the MC@NLO and POWHEG techniques are consistently implemented in the event generator SHERPA.

Summarising the above results, we find that the perturbative uncertainties associated with the POWHEG method are large, due to the unrestricted phase space available for exponentiation. It is important to stress that this problem is in principle solved by the MC@NLO method and that we simply use our implementation of MC@NLO to quantify the effect.

\subsection{Higgs-boson production in association with a jet}

This section presents predictions for the production of a Higgs boson in association with at least one jet. This process has not yet been investigated using either the Mc@NLO or the PowHEG approach. The Higgs mass is set to $m_{h}=120 \mathrm{GeV}$. Jets at partonlevel (i.e. before parton shower emissions take place) are defined using the inclusive $k_{\perp}$ algorithm [69-71] with $R=0.5$ and $p_{\perp, \min }=10 \mathrm{GeV}$. The independence of the results of the precise value of generation cut has been checked by varying $p_{\perp \text {, min }}=5 \ldots 15 \mathrm{GeV}$. Again, the effective coupling of the Higgs to gluons, mediated by a top-quark loop, is modeled through an effective Lagrangian [72, 73], and the virtual matrix elements here are computed by MCFM $[75,76]$. 
Both the renormalisation and factorisation scales are set to the transverse momentum of the hardest parton-level jet in the event. The two powers of $\alpha_{s}$ in the effective gluongluon-Higgs coupling are evaluated at a fixed scale $m_{h}$, independent of the renormalisation scale $\mu$. The reason for this choice is that scale variations of the effective coupling do not reflect a systematic uncertainty of the calculation at hand. They should be compensated by higher-order corrections to the effective coupling (i.e. diagrams with gluons attached to the top quarks in the loop), and will not be compensated by the one-loop corrections in the $h+$ jet matrix elements that we use. Thus, while the respective scale uncertainty is part of the systematics of the final cross section prediction, its proper assessment must come from a different calculation.

Our central prediction chooses $\alpha_{\text {cut }}=0.03$ to minimise the uncontrolled exponentiation of non-logarithmic terms and distortions of the Sudakov shape of the Higgs boson's transverse momentum spectrum. The predictions presented here include simulation of hadronisation [77, 78] and multiple parton interactions (MPI) $[79,80]$ as well as hadron decays [81] and higher-order QED corrections to the $h \rightarrow \tau \tau$ decay [82].

Uncertainty bands correspond to the variation of the renormalisation and factorisation scales in the range $1 / 2 \mu \ldots 2 \mu$ as well as to the variation of $\alpha_{\text {cut }}$ in a range from 1 to 0.001 . Scales are varied only in the matrix elements, not in the parton shower. As before, only minimal cuts are applied in the analysis: two $\tau$-leptons with $|\eta|<3.5$ and $p_{\perp}>25 \mathrm{GeV}$ are required. Jets are defined using the inclusive $k_{\perp}$-algorithm with $R=0.7$ and $p_{\perp, \min }=$ $20 \mathrm{GeV}$. Their minimum transverse momentum requested in the analysis ensures that the parton-level event selection is inclusive enough to guarantee coverage of the full phase space.

Figure 8 shows the transverse momentum spectra of the Higgs boson and the leading jet. Grey bands indicate the uncertainty due to the choice of $\alpha_{\text {cut }}$, which will be referred to as exponentiation uncertainty in the following. Yellow bands show the scale uncertainty. It is manifest, that the exponentiation uncertainty is largest in the region of large transverse momenta, a fact that seems counter-intuitive at first. Despite the transverse momentum distributions being described at NLO, they can apparently be altered significantly by additional emissions in the parton shower. Taking a closer look at eqs. (2.10)-(2.12), this fact becomes clear:

- The proof of next-to-leading order accuracy of both the POWHEG and the MC@NLO formulae is based on an expansion of the Sudakov form factors to first order. Higherorder corrections do play an important role, however, especially in regions of the phase space where parton emission is logarithmically enhanced. This region is drastically enlarged if the upper integration limit of the exponentiated real emission is shifted from $\mu_{F}$ to $s=E_{\mathrm{CMS}}^{2}$, as is the case in POWHEG and MC@NLO with $\alpha_{\text {cut }}=1$ (cf. section 4.1). This change alters the shape of the emission term in eq. (2.10) compared to the emission term in eq. (2.9). This finding is reinforced by the fact that in all distributions investigated, the predictions for $\alpha_{\text {cut }}=0.03$ coincide with those for $\alpha_{\text {cut }}=0.001$.

- The strong coupling is evaluated at a scale of the order of the relative transverse momentum between two partons in the emission term in eq. (2.9), while it is evaluated 

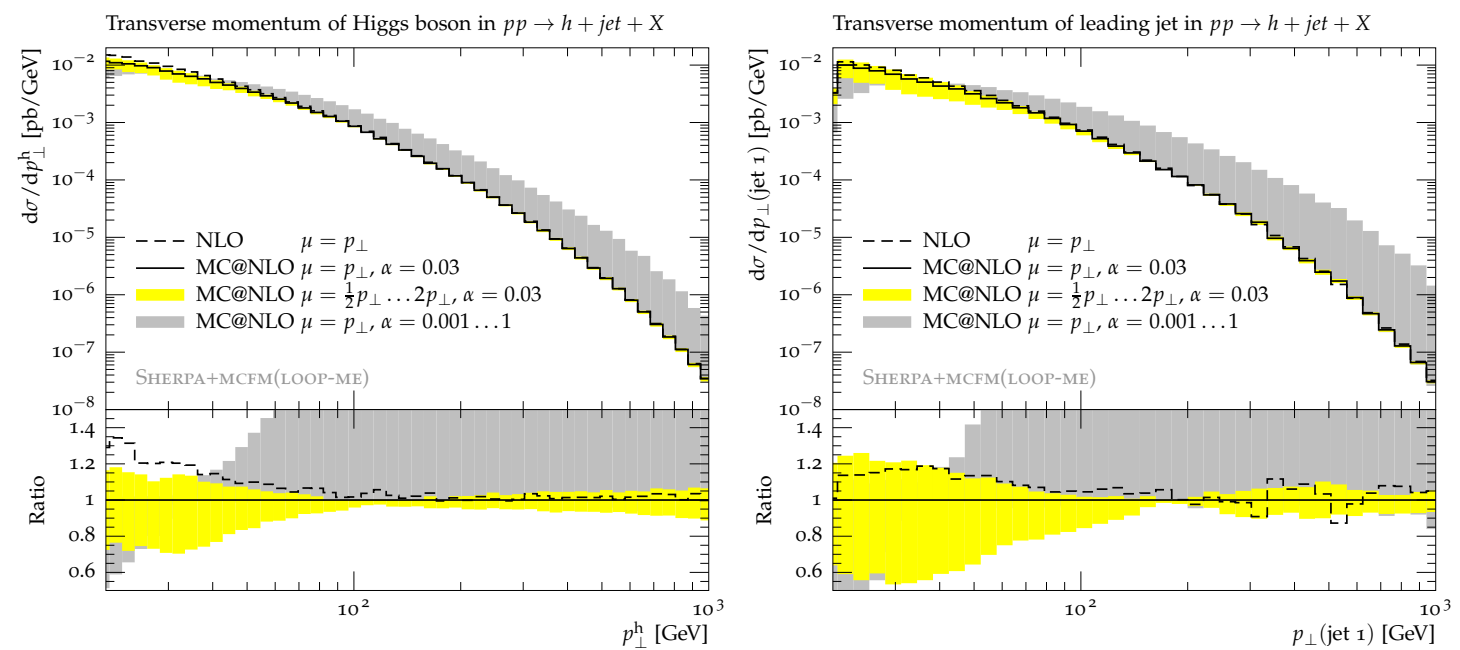

Figure 8. Prediction and uncertainties for the transverse momentum of the Higgs boson (left) and the leading jet (right) in Higgs boson plus jet events $\left(m_{h}=120 \mathrm{GeV}\right)$ at $E_{\mathrm{cms}}=7 \mathrm{TeV}$.
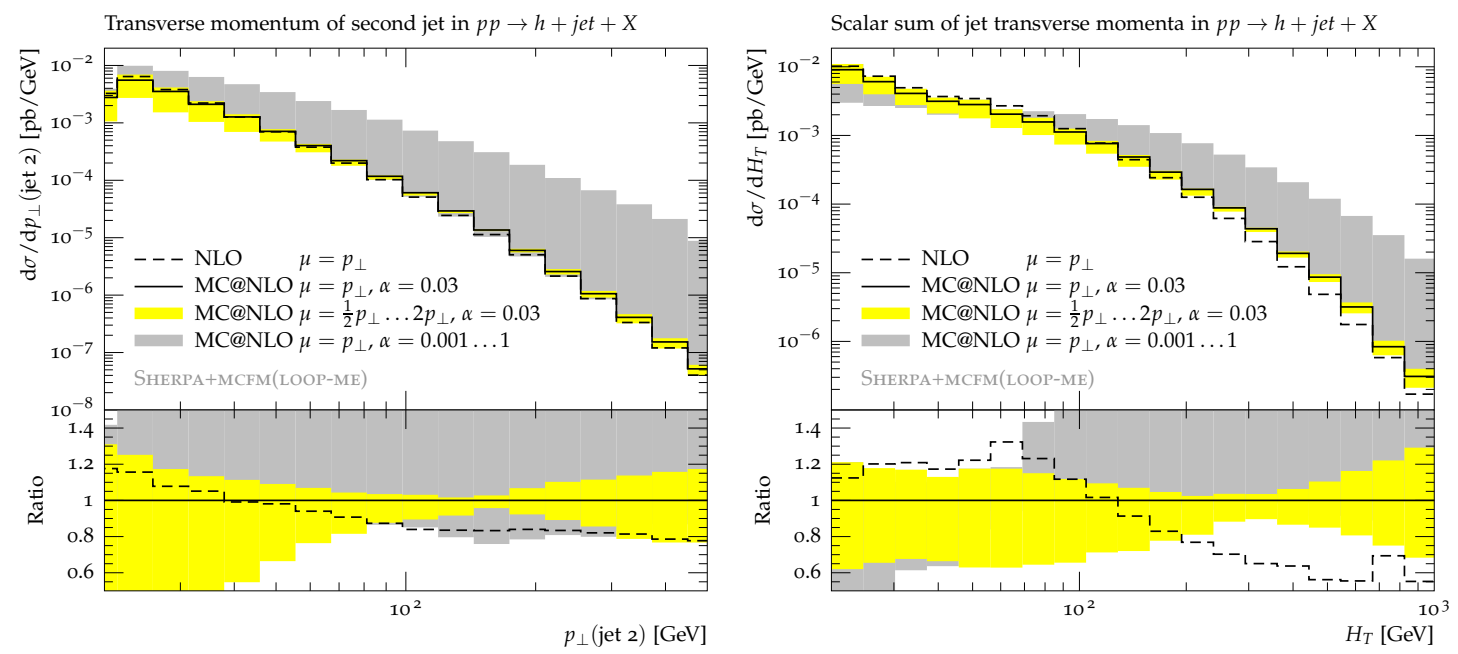

Figure 9. Predictions and uncertainties for transverse momentum of the second hardest jet (left) and the scalar sum of all jet transverse momenta (right) in Higgs boson plus jet events $\left(m_{h}=\right.$ $120 \mathrm{GeV}$ ) at $E_{\mathrm{cms}}=7 \mathrm{TeV}$.

at a different scale (here the transverse momentum with respect to the beam) in eq. (2.11). This leads to an additional distortion of the spectrum of the real-emission term, which is formally a sub-leading effect, but which becomes important in the enlarged logarithmically enhanced regions of the emission phase space.

The above effects are amplified in figure 9, as the observables there, the transverse momentum of the second jet and the scalar sum of all jets in the event, are described at leading-order accuracy only.

In contrast, figures 10 and 11 show a rather mild dependence on $\alpha_{\text {cut }}$ in the shape of the distributions. Nonetheless, a change in the normalisation can be observed, which is 

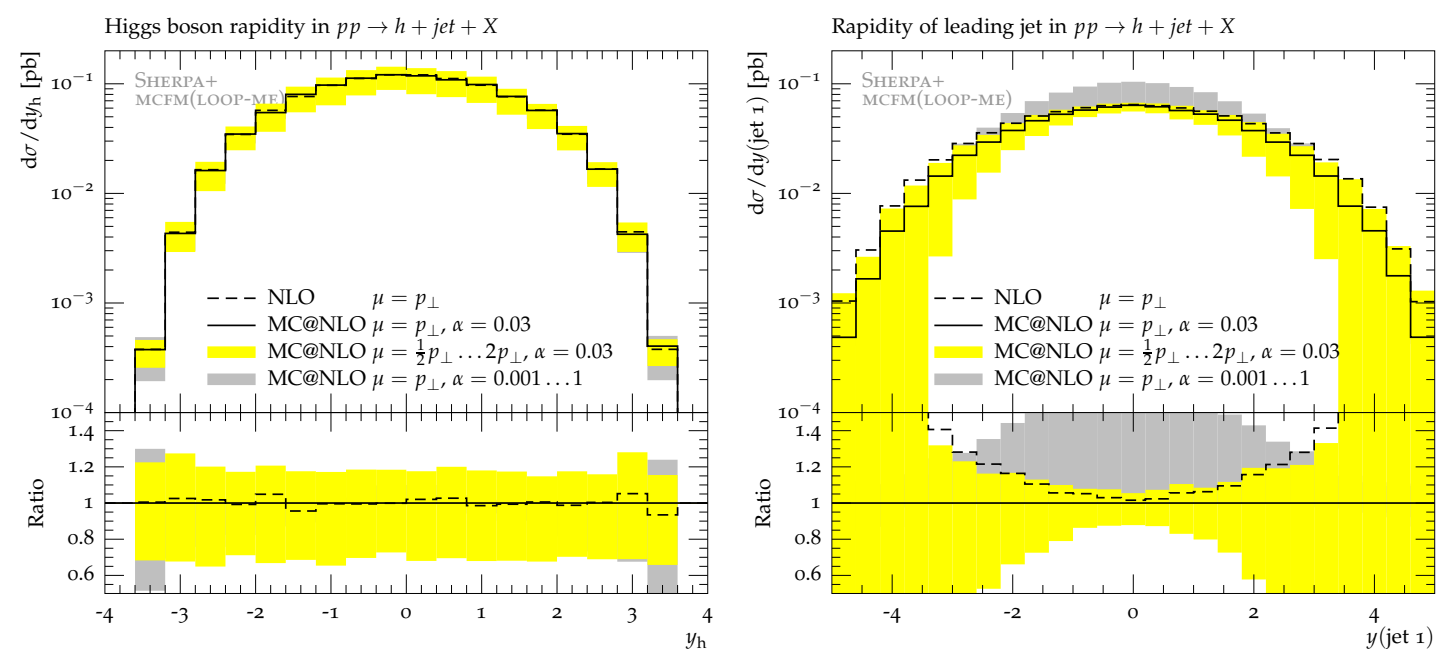

Figure 10. Prediction and uncertainties for the rapidity of the Higgs boson (left) and the leading jet (right) in Higgs boson plus jet events $\left(m_{h}=120 \mathrm{GeV}\right)$ at $E_{\mathrm{cms}}=7 \mathrm{TeV}$.
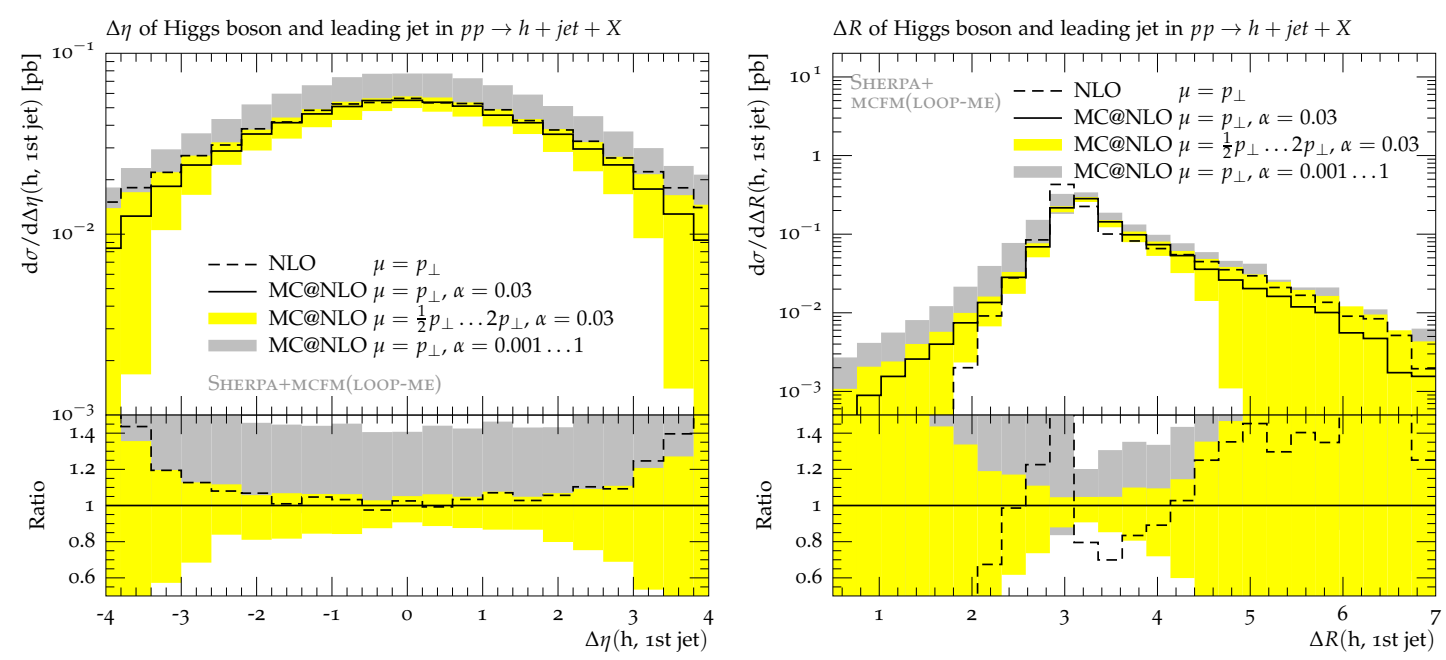

Figure 11. Predictions and uncertainties for the pseudorapidity (left) and angular (right) separation of the Higgs boson and the leading jet in Higgs boson plus jet events $\left(m_{h}=120 \mathrm{GeV}\right)$ at $E_{\mathrm{cms}}=7 \mathrm{TeV}$.

explained by the fact that due to larger emission rates with increasing $\alpha_{\text {cut }}$, the amount of radiative events is increased. Their kinematic distribution, however, does not seem to differ significantly. In fact, the large scale dependence observed in figures 8 and 9 is amplified here, especially for observables related to the hardest jet, indicating that, although $\mu=p_{\perp}$ is an appropriate scale choice for this process, the canonical variation to $\mu=\frac{1}{2} p_{\perp}$ leads to unphysical behaviour for jet rapidities beyond $|\eta| \approx 3.4$.

As a consequence of the strongly varying jet rates with varying $\alpha_{\text {cut }}$, the pseudorapidity separation between first and second hardest jet suffers from large uncertainties. It is displayed in figure 12. While the shape of the distribution is mostly unaffected, the normalisation varies strongly. The azimuthal separation between the two leading jets indicates 

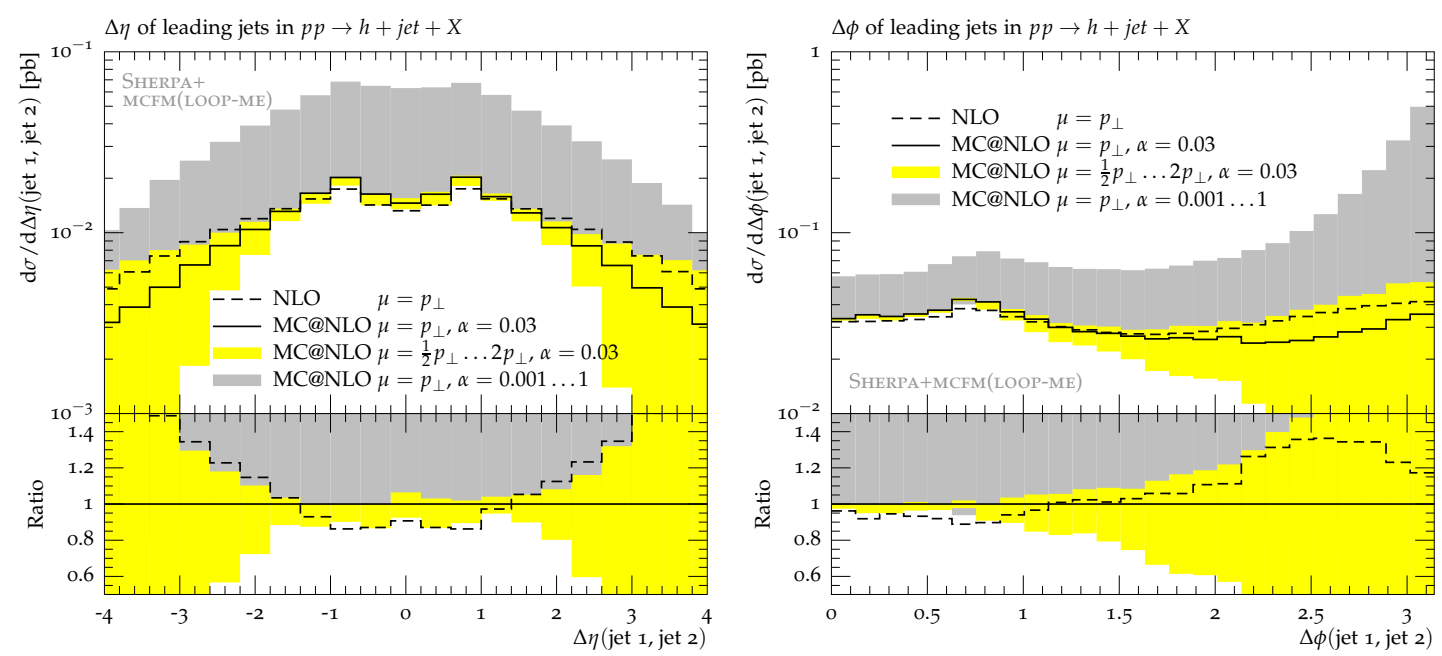

Figure 12. Predictions and uncertainties for the pseudorapidity (left) and azimuthal (right) separation of the two hardest jets in Higgs boson plus jet events $\left(m_{h}=120 \mathrm{GeV}\right)$ at $E_{\mathrm{cms}}=7 \mathrm{TeV}$.

how the exponentiation uncertainty affects the relative position of both jets in phase space. Compared to the pure next-to-leading order result, the back-to-back situation is amplified by a factor $\sim 10$ in the MC@NLO prediction. In such events, the Higgs-boson is likely to have been produced at $p_{\perp} \rightarrow 0$, consistent with the observation of a depletion of events in the first bins of the Higgs transverse momentum spectrum for $\alpha_{\text {cut }}=1$, cf. figure 8 .

\section{3 $W$-boson production in association with a jet}

In the following, $W[\rightarrow e \nu]+$ jet production in $p p$ collisions at $7 \mathrm{TeV}$ is studied. As for the case of Higgs-boson production, the MC@NLO implementation is validated against a fixedorder calculation. In addition the scale uncertainties are assessed by varying factorisation and renormalisation scales in MC@NLO by a factor of two in both directions.

To make the validation as meaningful as possible effects from parton showering beyond the first emission in $\mathbb{S}$-events, hadronisation and multiple parton scattering are not included in these parton level (PL) studies. Events are generated requiring at least one jet with $p_{\perp, \min }=10 \mathrm{GeV}$, defined according to the inclusive $k_{\perp}$-algorithm [69-71] with $R=0.5$. The transverse momentum of that hardest jet also sets the central value of factorisation and renormalisation scales. The level of exponentiation is fixed by $\alpha_{\text {cut }}=0.03$.

The total generated cross sections of the NLO calculation, $\sigma_{\mathrm{NLO}}=(4695 \pm 12) \mathrm{pb}$, and $\mathrm{MC} @ \mathrm{NLO}, \sigma_{\mathrm{MC} @ \mathrm{NLO}}=(4680 \pm 30) \mathrm{pb}$, agree within statistical uncertainties. The total generated cross section in MC@NLO varies between $\sigma(\mu / 2)=(4950 \pm 40) \mathrm{pb}$ and $\sigma(\mu \cdot 2)=(4510 \pm 23) \mathrm{pb}$, i.e. up to $5.8 \%$ around the central value.

Properties of the $W$-boson, which is reconstructed from the truth neutrino and the lepton, are displayed in figure 13. Agreement between fixed-order and MC@NLO results in both the rapidity and transverse momentum spectra is found. Agreement is also found for the transverse momentum and pseudorapidity distributions of the electron, which are shown in figure 14 . 

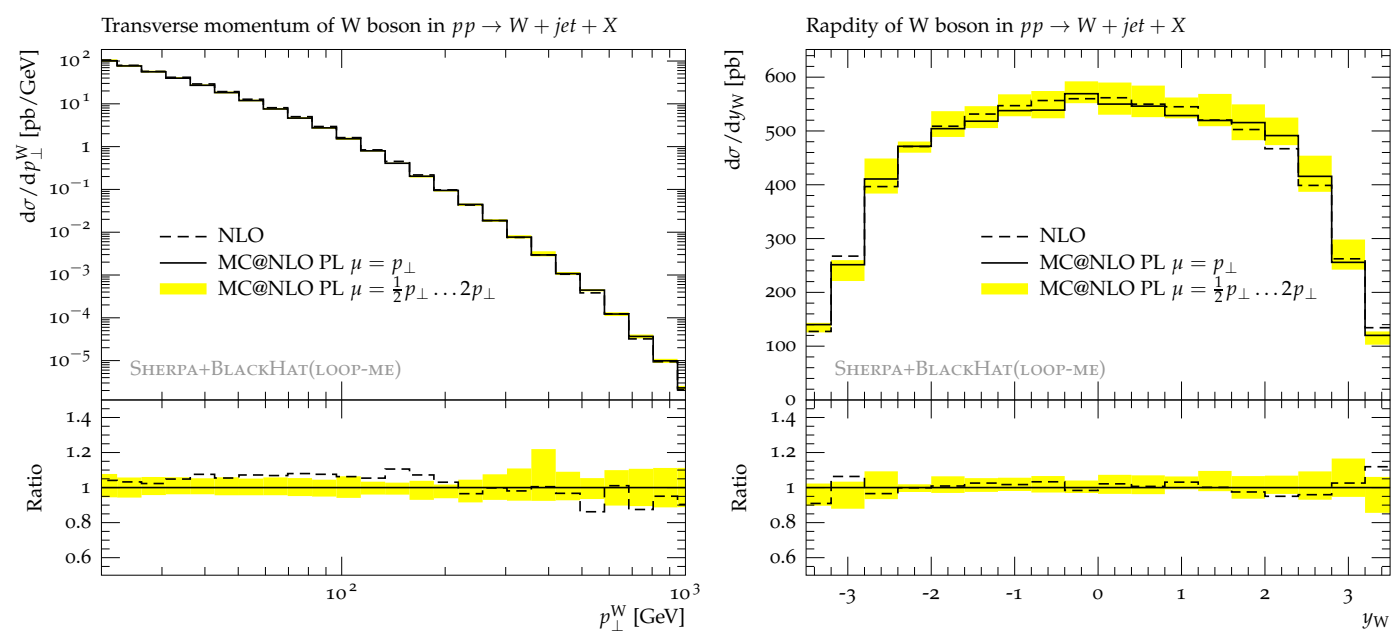

Figure 13. Transverse momentum and rapidity of the $W$-boson in $W[\rightarrow e \nu]+j$ production at the LHC.
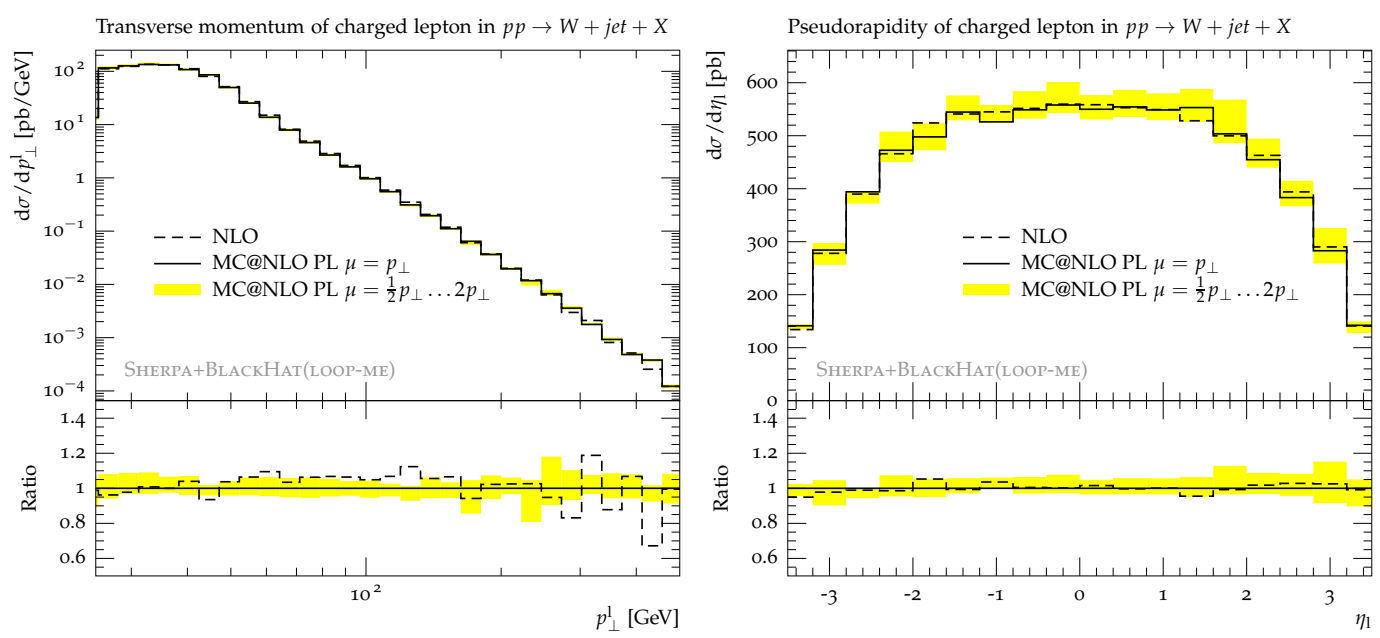

Figure 14. Transverse momentum and pseudorapidity of the electron in $W[\rightarrow e \nu]+j$ production at the LHC.

In the event analysis jets are defined according to the inclusive $k_{\perp}$ algorithm [69-71] with $R=0.7$ and requiring $p_{\perp}>20 \mathrm{GeV}$. Properties of these jets are displayed in figure 15 . The transverse momentum of the leading jet, an observable described at next-to-leading order accuracy, shows good agreement between both approaches. The scalar sum of jet transverse momenta $\left(H_{T}\right)$ is only described at leading-order accuracy and thus suffers from larger exponentiation uncertainties. This leads to a disagreement between the fixed-order result and MC@NLO, up to the level of $20 \%$.

No distribution is affected significantly by scale variations beyond the change in total rate. This indicates that the functional form of the scale choice described at the beginning of this section works well for this process. 

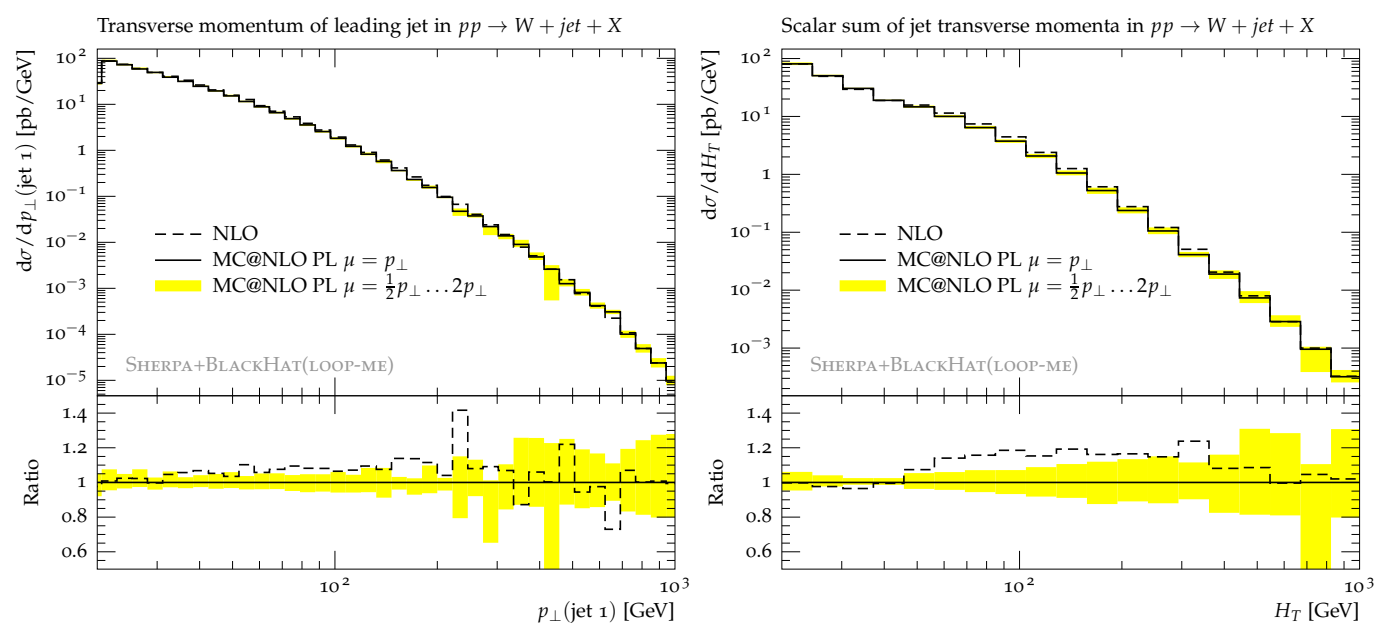

Figure 15. Transverse momentum of the leading jet and scalar sum of all jet transverse momenta in $W[\rightarrow e \nu]+j$ production at the LHC.

\section{Non-perturbative uncertainties and comparison to data}

In this section the MC@NLO method is further studied using $W+$ jet and $Z+$ jet production as a testing ground. Emphasis is now placed on the investigation of non-perturbative effects and associated systematic variations.

\subsection{Analysis of non-perturbative effects}

The Mc@NLO method allows to generate fully hadronised events as an input for detector simulation or for direct comparison to measurements at the particle level. A question that naturally arises is, whether the theoretical uncertainties of the full MC@NLO simulation are then dominated by the perturbative or non-perturbative effects. We do not attempt to judge on this question here as it is not obvious on which grounds they can be compared at all, but rather point out that both non-perturbative corrections and non-perturbative uncertainties are modest compared to the intrinsic uncertainties of the parton-level result, which were investigated in the previous section.

To this end, MC@NLO simulations for $W+$ jet production are compared with a varying level of non-perturbative effects included:

\section{"Parton Level"}

Only the first emission off $\mathbb{S}$-events in MC@NLO is generated in addition to the seed event. This is the same method that was used in the comparison to fixed-order results in section 4.3 .

\section{"Shower Level"}

All QCD emissions in the parton shower and QED emissions in the YFS approach are taken into account. 

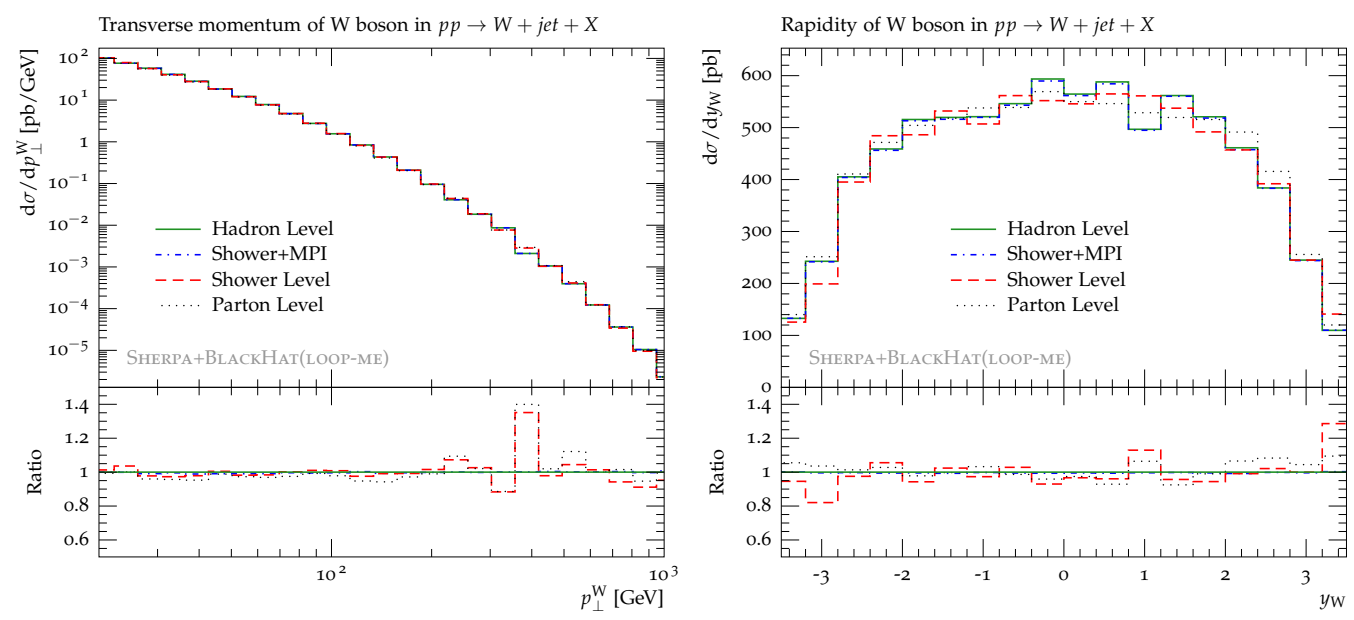

Figure 16. Transverse momentum and rapidity spectrum of the $W$-boson in $W[\rightarrow e \nu]+j$ production at the LHC.
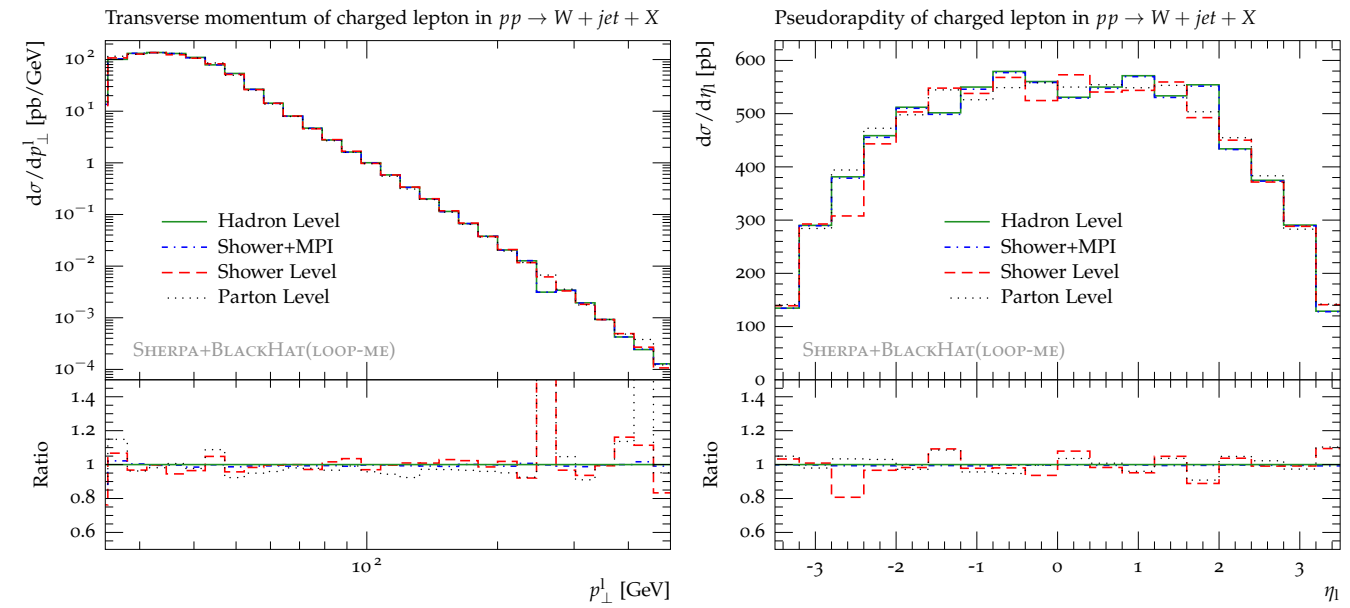

Figure 17. Transverse momentum and pseudorapidity spectrum of the electron in $W[\rightarrow e \nu]+j$ production at the LHC.

\section{"Shower+MPI"}

Multiple parton interactions and intrinsic transverse momentum of the beam hadron are additionally allowed for.

\section{"Hadron Level"}

Hadronisation and hadron decays are included to generate events at the particle level.

All other event generation parameters have been chosen identical to section 4.3.

Non-jet observables, like the rapidity and transverse momentum of the $W$-boson and the rapidity and transverse momentum of the charged lepton are virtually unaffected by non-perturbative effects, as expected. This is shown in figures 16 and 17.

Jet observables are far more sensitive, as is exemplified by the transverse momentum and rapidity spectra of the leading and next-to-leading jet in figure 18. They show effects of 

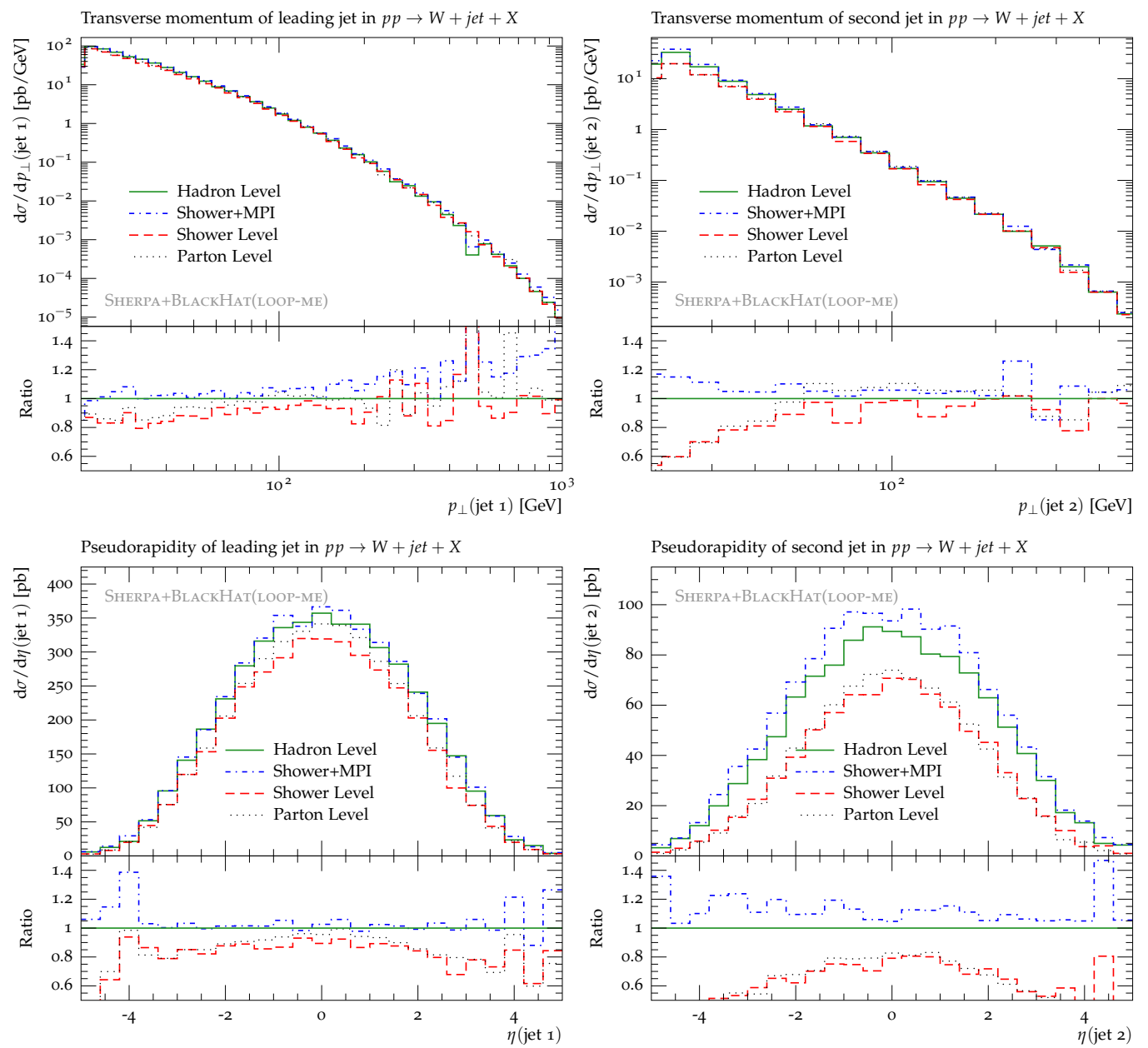

Figure 18. Transverse momenta and pseudorapidities of the two leading jets in $W[\rightarrow e \nu]+j$ production at the LHC.

"out-of-cone" radiation at shower level, which are partially compensated by the simulation of multiple scattering effects. Hadronisation again leads to softer jet spectra, such that the various effects can compensate each other. Although the tendency of the corrections stays the same, their precise magnitude depends on the jet algorithm and its parameters and will have to be investigated separately for each analysis. Figure 19 exemplifies how non-perturbative effects can distort correlations between the two hardest jets.

The uncertainties inherent in the hadronisation model were probed by switching from the SHERPA default cluster fragmentation model $[77,78]$ to the Lund string model $[83,84]$ in the implementation of PYTHIA [62]. Both models have been tuned to data from LEP and excellent agreement has been achieved. For this comparison to be meaningful the same perturbative events (i.e. identical random seeds) were subjected to the two hadronisation models. Effects from statistical fluctuations are thus cancelled.

The differences were found to be negligible for the observables studied here, as displayed in figure 20, except for the specifically hadronisation-sensitive jet mass, where variations up to $20 \%$ occur. 

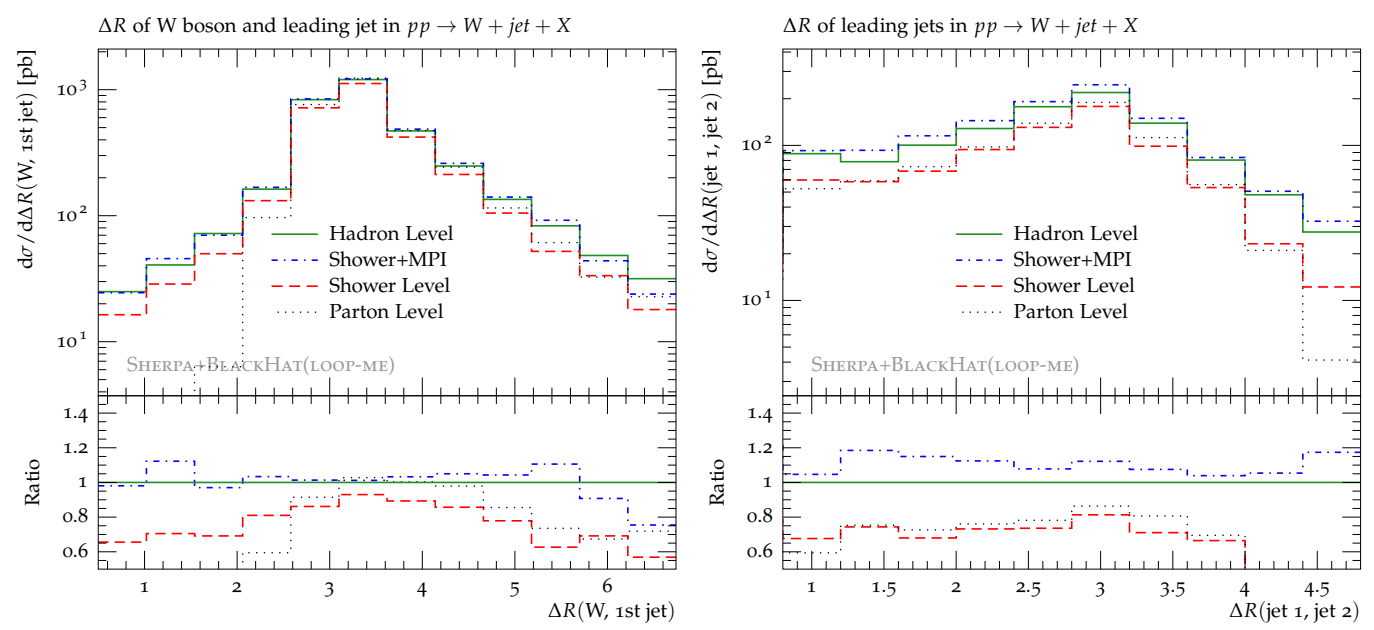

Figure 19. Correlations between $W$-boson and leading jets in $W[\rightarrow e \nu]+j$ production at the LHC.

\section{$5.2 Z+$ jet production compared to Tevatron data}

Let us now turn to the comparison of MC@NLO predictions to data and a simple assessment of the systematic uncertainties associated with a significant part of the non-perturbative effects. Central predictions are made at the hadron level with SHERPA's default tune. An uncertainty band is generated by allowing for a variation of the MPI parameters within boundaries given by existing measurements of the underlying event. All event generation parameters have been chosen analogous to the $W+$ jet case above.

Figure 21 compares $Z+$ jet production in the electron channel to a measurement from the CDF collaboration [85]. The reconstructed electrons are required to have transverse momenta $p_{\perp}>25 \mathrm{GeV}$ and an invariant mass of $66<m_{e e}<116 \mathrm{GeV}$. Jets are defined using the midpoint cone algorithm [86] with $R=0.7$ and a split/merge fraction of 0.75 . At least one jet with $p_{\perp}>30 \mathrm{GeV}$ and $|y|<2.1$ needs to be present and separated from both electrons by $\Delta R_{e j}>0.7$. The cross section in the one-jet bin is predicted slightly too low. The cross section of the two-jet bin is significantly underestimated, as it is determined to leading order accuracy and subject to large uncertainties from the NLO+PS matching procedure as discussed in section 4 . The shape of the transverse momentum distributions agrees well with data.

More characteristics of $Z$-boson plus jet production were investigated in a recent $\mathrm{D} \varnothing$ analysis [87]. Events with two muons of invariant mass $65<m_{\mu \mu}<115 \mathrm{GeV}$ and with at least one jet of $p_{\perp}>20 \mathrm{GeV}$ and $|y|<2.8$ were collected at a center-of-mass energy of $1.96 \mathrm{TeV}$. Jets were defined using the $\mathrm{D} \varnothing$ midpoint cone algorithm [88] with $R=0.5$ and a split/merge fraction of 0.5 . Each jet had to be separated from both leptons by $\Delta R_{\mu j}>0.5$. A comparison of Monte-Carlo predictions with this measurement is shown in figure 22. The agreement is fair, except for the $p_{\perp}$-spectrum of the first jet, where a deficiency of the Monte-Carlo result at intermediate and high $p_{\perp}$ is observed.

A further measurement of $Z+$ jet production in the electron channel was presented by the $\mathrm{D} \varnothing$ collaboration in [89]. Each electron is required to have $p_{\perp}>25 \mathrm{GeV}$ and 

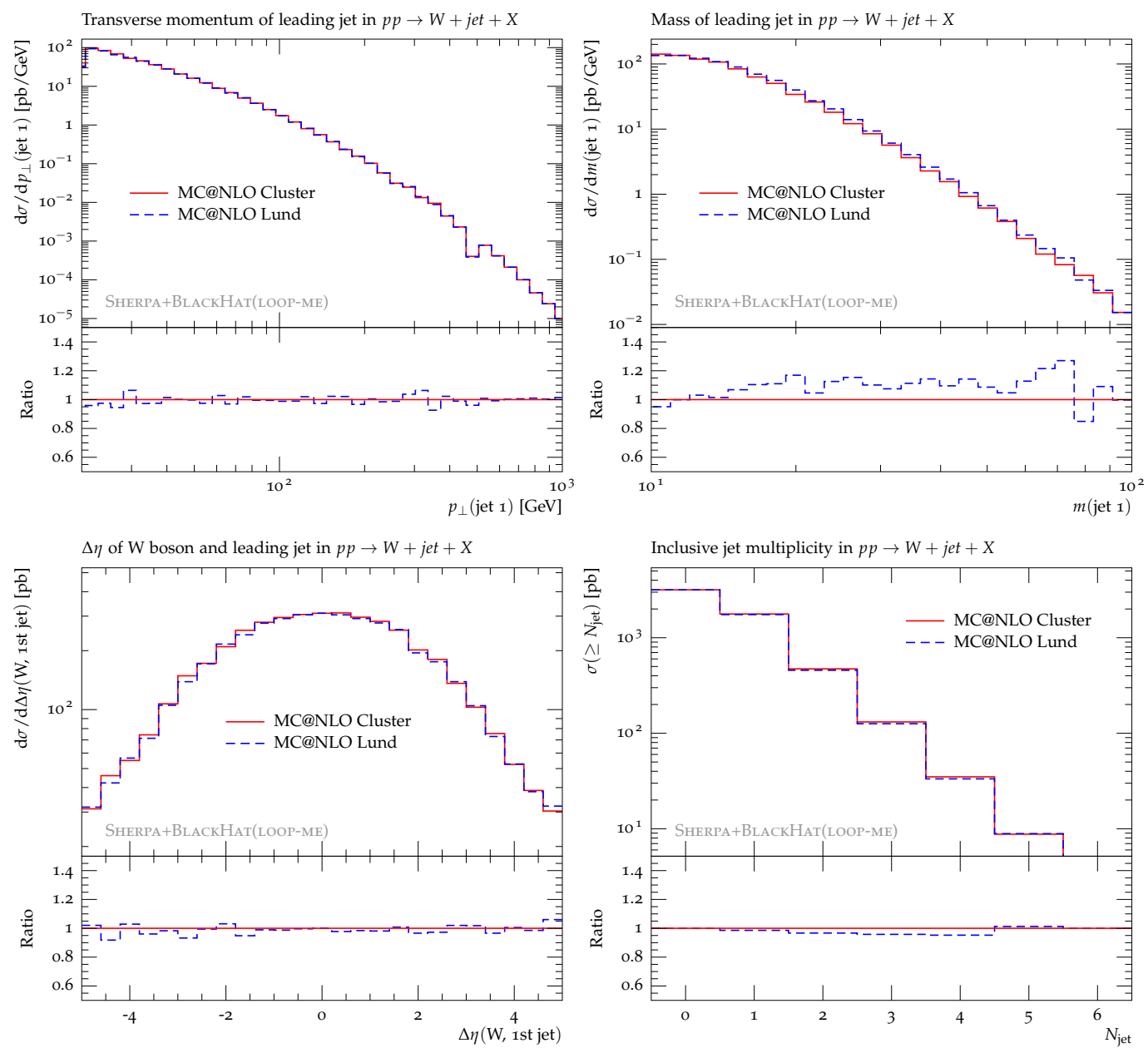

Figure 20. Hadronisation uncertainties for different observables studied in $W[\rightarrow e \nu]+j$ production at the LHC.
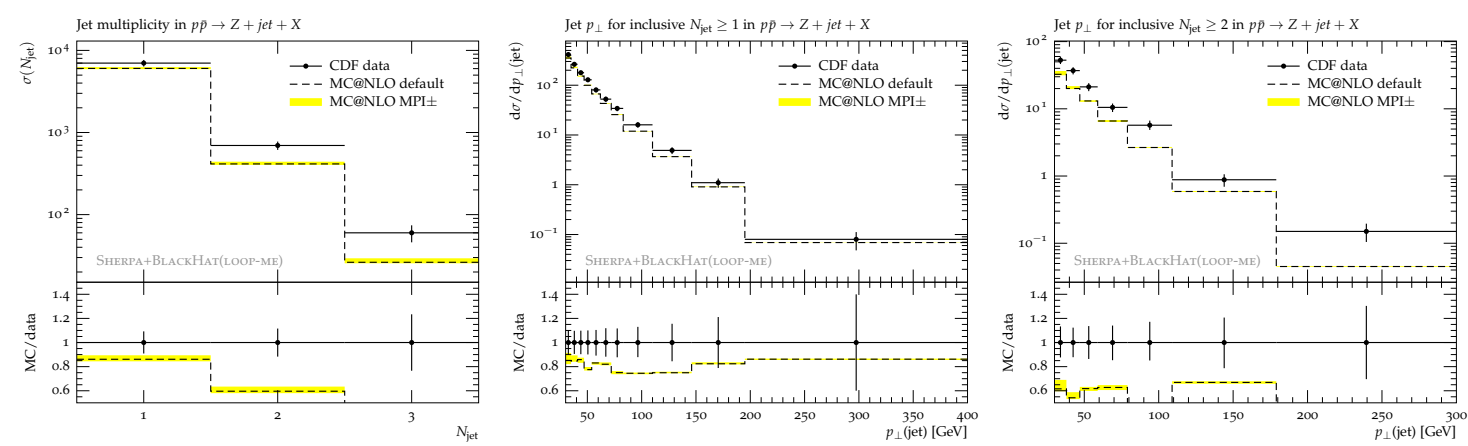

Figure 21. Inclusive jet cross sections and inclusive transverse momentum distributions of all and all-but-the-hardest jets compared to CDF data [85].

the mass window $65<m_{e e}<115 \mathrm{GeV}$ is enforced. Jets are defined using the midpoint cone algorithm [88] with $R=0.5$ and a split/merge fraction of 0.5. At least one jet with $p_{\perp}>20 \mathrm{GeV}$ and $|\eta|<2.5$ must be present in the event. Experimental data were 

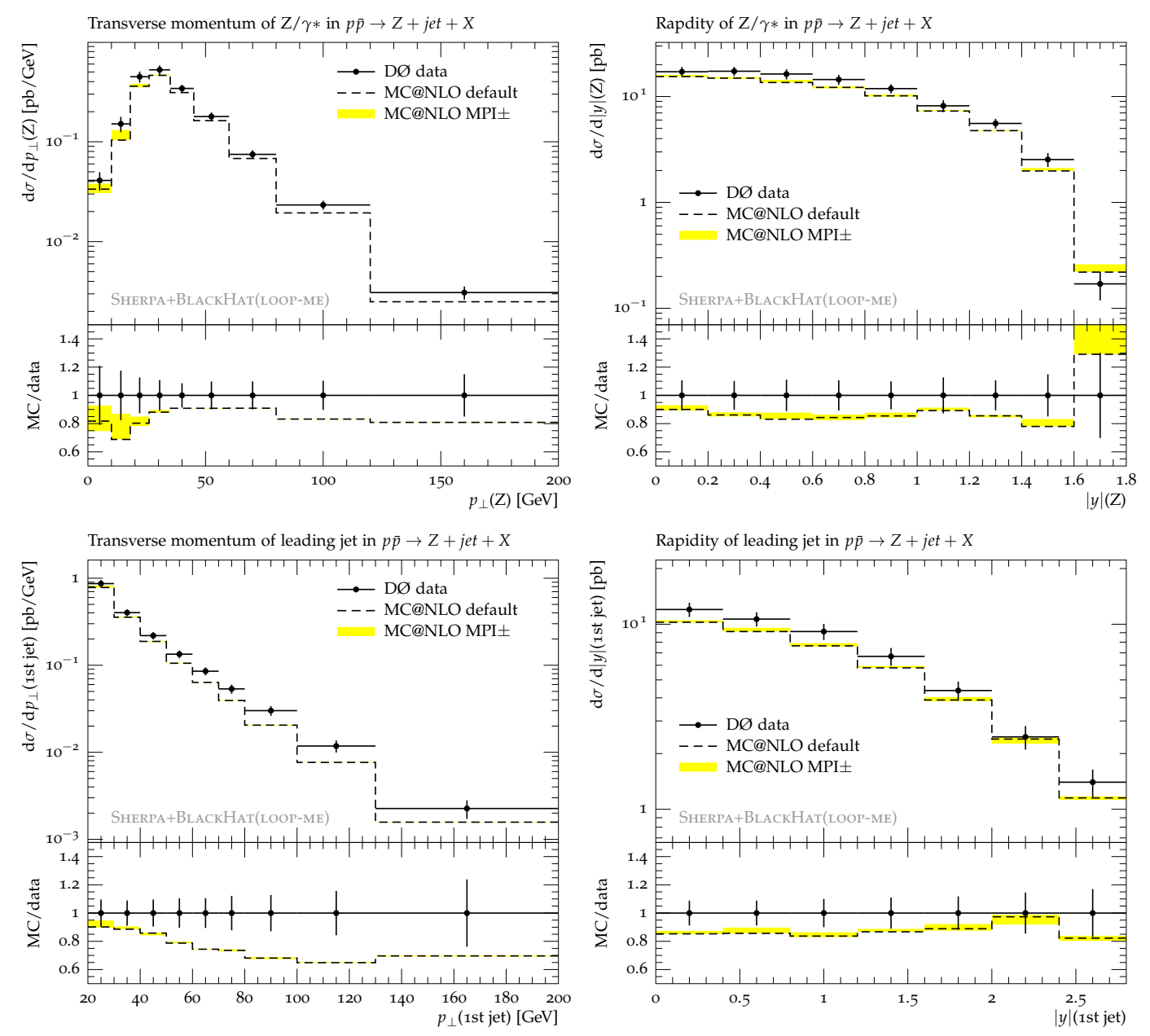

Figure 22. Transverse momentum and rapidity distributions of the reconstructed $Z$ boson (top row), and the total cross section, the transverse momentum and rapidity distributions of the hardest jet (bottom row) in Drell-Yan production in association with at least one jet compared to DØ data [87].

normalised to the inclusive Drell-Yan cross section. This quantity is not predicted by the MC@NLO simulation of $Z+$ jet, and therefore the MC@NLO results are scaled by a global factor of 0.35 such that the normalisation of the one-jet-rate agrees with data. Figure 23 displays the comparison of these scaled MC@NLO predictions with the results from DØ. The transverse momentum shape of the leading jet is well described by the simulation. The two-jet rate, described here at leading-order accuracy, seems to be underestimated compared to data, but the shape of the sub-leading jet's $p_{\perp}$ spectrum is relatively flat. For the third jet, which is generated in the parton-shower approximation, both the rate and the shape of the spectrum are not simulated correctly.

To quantify the success of the next-to-leading order calculation it is important not only to investigate single-particle spectra, but also correlations between the $Z$-boson and the hardest jet. They might give some insight into the genuine one-loop effects in $Z+$ jet production. Therefore, the analysis strategy of a measurement presented by the $\mathrm{D} \varnothing$ collab- 

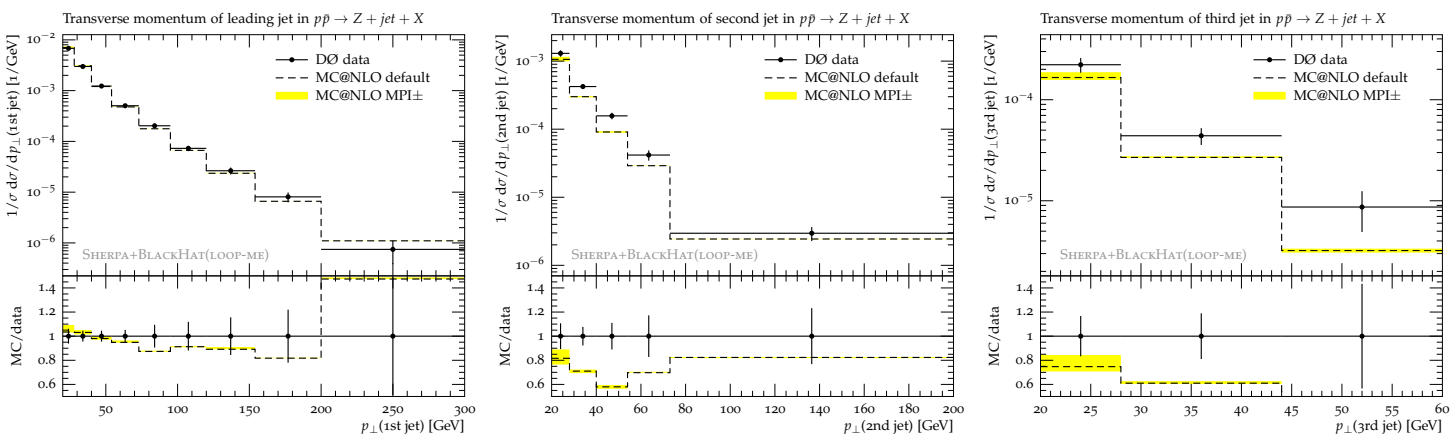

Figure 23. Transverse momentum distributions of the three hardest jets in Drell-Yan production in association with at least one jet compared to D $\varnothing$ data [89]. All SHERPA predictions are scaled with a common factor to account for the unknown normalisation to the inclusive process.
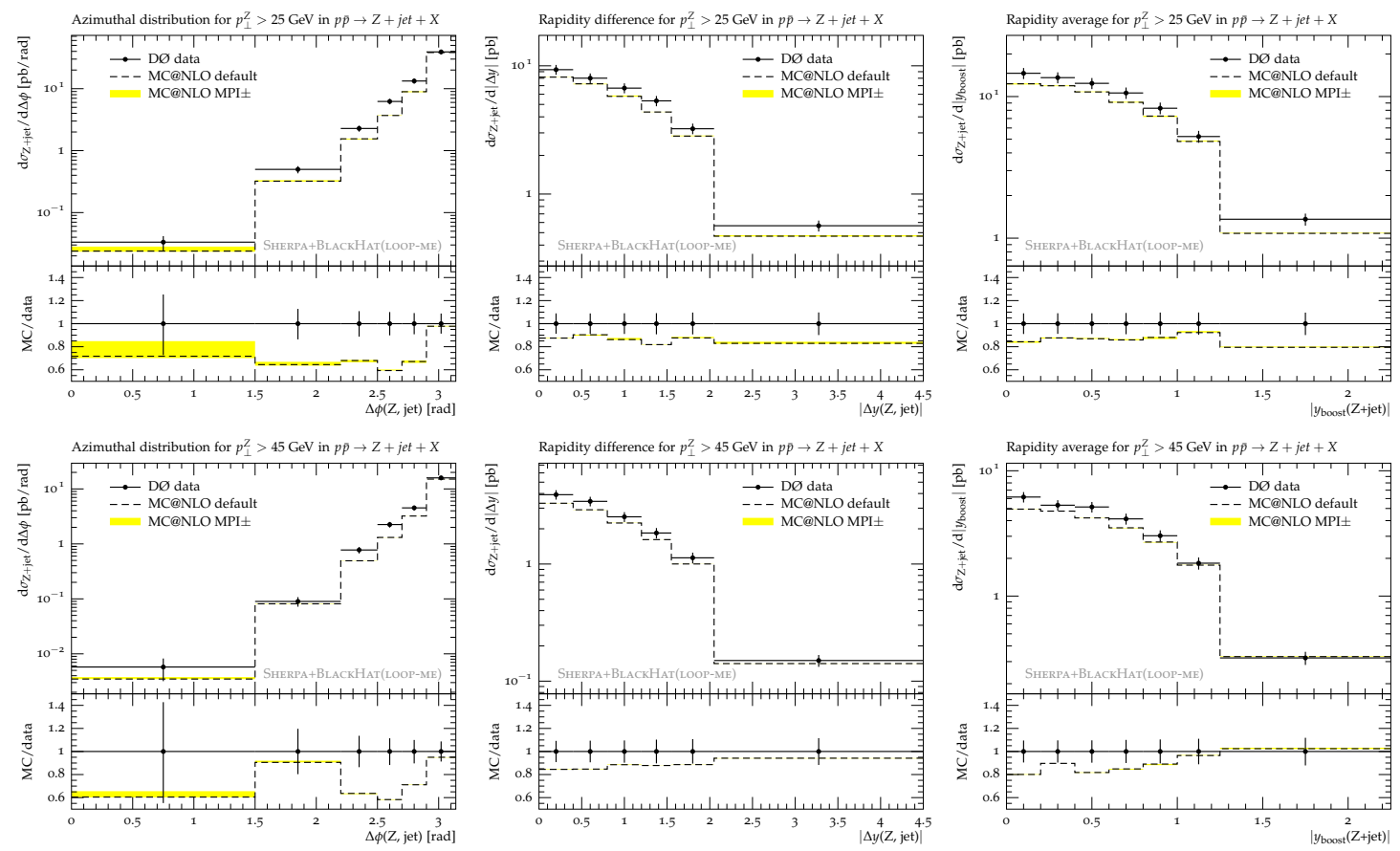

Figure 24. Azimuthal and rapidity difference distributions of the reconstructed $Z$ boson and the hardest jet and their rapidity average in Drell-Yan production in association with at least one jet compared to DØ data [90].

oration in [90] is pursued. Opposite-sign muons with $p_{\perp}>15 \mathrm{GeV}$ and an invariant mass of $65<m_{\mu \mu}<115 \mathrm{GeV}$ are required in association with at least one jet of $p_{\perp}>20 \mathrm{GeV}$ and $|y|<2.8$. Jets are defined using the midpoint cone algorithm [88] with $R=0.5$ and a split/merge fraction of 0.5 . Two event samples are defined, one requiring the transverse momentum of the reconstructed $Z$ boson to be above $25 \mathrm{GeV}$, the other requiring it to be above $45 \mathrm{GeV}$. Figure 24 compares Mc@NLO predictions to the measurement. The shape of the rapidity distributions is matched fairly well, again with the total rate prediction at the lower end of the uncertainty band. For the azimuthal correlation distribution 

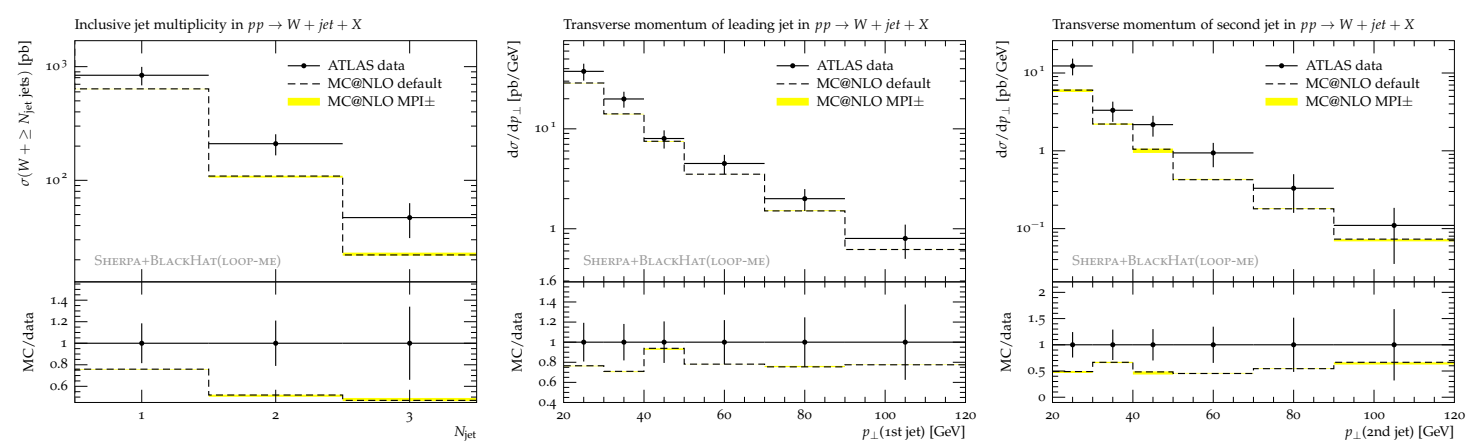

Figure 25. Inclusive jet cross sections and transverse momentum spectra of the hardest and second hardest jet in $W[\rightarrow e \nu]+j$ production at the LHC compared to ATLAS data [27].

the shape shows significant deviations from data: Only the back-to-back configuration is described well, but for $\Delta \phi<\pi$ the MC@NLO prediction underestimates the data. This signals the uncertainty related to emissions beyond the hardest one, which are only generated at leading-order or parton-shower accuracy and which are also subject to large NLO+PS matching systematics as discussed in section 4 .

\section{3 $W+$ jet production compared to LHC data}

The production of a $W$ boson in association with at least one hard jet in proton-proton collisions at $\sqrt{s}=7 \mathrm{TeV}$ has been studied by the ATLAS collaboration at the CERN LHC [27].

In the electron channel events are selected by requiring an electron with $p_{\perp}>20 \mathrm{GeV}$ defined at the particle level to include all photon radiation within a $\Delta R=0.1$ cone. Only electrons in the fiducial volume $|\eta|<1.37$ or $1.52<|\eta|<2.47$ are taken into account. $E_{\perp}^{\text {miss }}$ at the particle level has been defined through the leading neutrino in the event which is required to have $p_{\perp, \nu}>25 \mathrm{GeV}$. The transverse mass cut is placed at $m_{T}=\sqrt{2 p_{\perp}^{\ell} p_{\perp}^{\nu}\left(1-\cos \left(\phi_{\ell}-\phi_{\nu}\right)\right)}>40 \mathrm{GeV}$.

Jets are reconstructed using the anti- $k_{t}$ algorithm with $R=0.4$ and have been taken into account if $p_{\perp}^{\text {jet }}>20 \mathrm{GeV},\left|\eta_{\text {jet }}\right|<2.8$ and $\Delta R(\ell$, jet $)>0.5$. Muons, neutrinos and the leading electron were excluded from the input of jet reconstruction.

The comparison in figure 25 shows good agreement of the SHERPA hadron level prediction with ATLAS data in the shapes of differential distributions on the one hand, and discrepancies in the prediction of the total rate on the other hand. While the one-jet rate is still predicted fairly well, the two and three-jet rates are significantly too low. The latter are only predicted at leading-order and parton-shower accuracy and also subject to the inherent uncertainties from NLO+PS matching.

\section{Conclusions}

In this publication, the MC@NLO and POWHEG methods to match NLO QCD matrix elements with parton showers have been compared, with a special emphasis on some issues 
that generate large differences between their predictions. In particular, these issues are related to

- the treatment of sub-leading colour configurations in MC@NLO

- the choice of scales in POWHEG and MC@NLO and

- the exponentiation of non-leading terms in POWHEG.

Before discussing the findings related to these issues, it is worth summarising some other results presented in this publication. They refer to the impact of scale variations at the parton-level of the processes studied here, and to the effect of hadronisation and the underlying event on a number of observables:

- Scale uncertainties

In $W / Z(+$ jets $)$ production, uncertainties due to scale variations are typically about $5-10 \%$, but they can increase up to about $25 \%$ in the high- $p_{\perp}$ region of jet production, where higher jet multiplicities become relevant. In Higgs production processes scale uncertainties tend to be significantly larger, by at least a factor of 2 , which is indicative of large higher-order corrections.

- Underlying event corrections and uncertainties

Multiple parton interactions tend to increase jet production rates, and they have a particularly large influence on observables which are sensitive to high jet multiplicities, like $H_{T}$. They typically do not affect inclusive observables, with changes of about $5 \%$ or below. Their associated uncertainty is small.

- Hadronisation corrections and uncertainties

Hadronisation corrections are similar in magnitude to parton-shower and multiplescattering effects. The associated uncertainties are about $5 \%$, except for observables such as jet masses, where they can increase to about $20 \%$.

Let us now turn to the uncertainties induced by the NLO+PS matching.

Regarding the problem of infrared divergences in sub-leading colour configurations, it should be noted that up to now, only one critical process - heavy quark pair production has been implemented in a publicly available and fully documented event generator. In this case the problem was solved by introducing a factor that modifies the integral in eq. (2.11) such that contributions from the region of soft-gluon emission are removed. A similar strategy seems to be employed in the recently presented aMC@NLO code, but it has not been published or made publicly available yet. It is therefore not yet clear whether this technique is sufficiently process-independent to allow for a general implementation of MC@NLO. In this publication a different approach has been followed, which aims instead at constructing an exact solution, irrespective of the process considered. The basic idea is to correct the Casimir operators used in the parton shower such that the full colour structure in the soft-gluon limit is obtained. This introduces both positive and negative splitting functions, which is unproblematic for NLO calculation but implies "anti-probabilistic" evolution in 
a parton shower. To resolve this issue, a weighting procedure has been introduced. It is anticipated that this modification will eventually allow the systematic inclusion of subleading colour terms in the parton shower.

The exponentiation of non-leading terms in POWHEG is an issue that has been known for some time. Actual differences in how POWHEG and MC@NLO predict the $p_{\perp}$ spectrum of the Higgs boson are documented in the literature [56]. They have typically been attributed to a different treatment of higher order, in particular NNLO, corrections (see section 4.3 of [56]). We find that the difference is related to exponentiation uncertainties, naturally affecting the next-to-next-to leading order, but continuing to all orders. The upper scale in the logarithms resummed in POWHEG is related to the total hadronic centre-of-mass energy, which is substantially different from the scale typically used in the resummation programme [91, 92], where a value of the order of the Higgs boson mass is assumed. It is thus clear that a suitable constraint on the emission phase space in POWHEG must be defined. This problem has already been solved by the MC@NLO method. On a related note, the occurrence of the well-known dip around zero in the MC@NLO simulation of the $y_{j}-y_{H}$ distribution, the rapidity difference of Higgs boson and accompanying jet, has been studied here. Cutting on the phase space of the Catani-Seymour subtraction kernels and reflecting these cuts in the parton shower, as enforced by MC@NLO, yields dead zones of parton emission in the latter. While these dead zones are clearly different from the dead zones in the HERWIG parton shower, the resulting effect is surprisingly similar. In fact, both including the full phase space and comparably tight cuts on the phase space leads to a vanishing dip; this finding suggests that the dip, which is not present in the NLO calculation, must be attributed to true exponentiation uncertainties.

To complete our implementation of the MC@NLO method in the sense that only logarithms related to emissions on scales below the factorisation scale are exponentiated, physically more meaningful cuts on the phase space of Catani-Seymour subtraction kernels are currently investigated. Devising a similar technology for PowHEG would result in two formally completely equivalent algorithms, but imply a larger computational effort for event generation in POWHEG due to the additional numerical integration in $\bar{B}$ and the matrix-element correction of the weighted parton shower, see [40]. For this reason, in favour of the MC@NLO method, the further development of POWHEG methods in the SHERPA framework will be abandoned.

\section{Acknowledgments}

SH's work was supported by the US Department of Energy under contract DE-AC0276SF00515, in part by the US National Science Foundation, grant NSF-PHY-0705682, (The LHC Theory Initiative), and in part by the US National Science Foundation under grant NSF-PHY-0551164. MS's work was supported by the Research Executive Agency (REA) of the European Union under the Grant Agreement number PITN-GA-2010-264564 (LHCPhenoNet). FS's work was supported by the German Research Foundation (DFG) via grant DI $784 / 2-1$. 
The authors would like to thank Massimiliano Grazzini and Stefano Catani for fruitful discussion. FK would also like to thank the Galileo Galilei Institute in Florence and the SLAC theory group for their hospitality while this work was finished. SH thanks the $\mathrm{CP}^{3}$-Origins Odense for hospitality.

\section{A Comment on the NLL accuracy of the POWHEG formula}

In [93] it was proven that the POWHEG formula yields NLL accurate predictions, if the strong coupling is modified according to [94]. The proof rests on the crucial assumption that when logarithms of the form $L=\log \left(p_{\perp} / Q\right)$ are resummed, the dependence on the resummation scale $Q$ introduces corrections beyond next-to-leading logarithmic order. This argument holds as long as $Q$ differs from the hard scale in the process, say $m_{h}$ in Higgsboson production, by a factor of order one [91, 92, 95-97]. As pointed out in [91] large spurious subleading logarithms may otherwise be introduced, that are associated with the overall normalization of observables, rather than radiative corrections. In other words, in the large- $k_{T}$ region the resummed results lose their predictivity and should be replaced by conventional fixed-order calculations [97]. Following these arguments, the reasoning in [93] is only correct as long as the resummation scale and the hard scale are sufficiently similar. This is not the case in the POWHEG method when it is applied to hadronic collisions where $Q \rightarrow E_{\text {cms }}$, the total hadronic centre-of-mass energy, while the hard scale is of order $m_{h}$, for example.

Open Access. This article is distributed under the terms of the Creative Commons Attribution License which permits any use, distribution and reproduction in any medium, provided the original author(s) and source are credited.

\section{References}

[1] S. Catani, F. Krauss, R. Kuhn and B. Webber, QCD matrix elements + parton showers, JHEP 11 (2001) 063 [hep-ph/0109231] [INSPIRE].

[2] M.L. Mangano, M. Moretti and R. Pittau, Multijet matrix elements and shower evolution in hadronic collisions: $W b \bar{b}+n$ jets as a case study, Nucl. Phys. B 632 (2002) 343 [hep-ph/0108069] [INSPIRE].

[3] F. Krauss, Matrix elements and parton showers in hadronic interactions, JHEP 08 (2002) 015 [hep-ph/0205283] [INSPIRE].

[4] L. Lönnblad, Correcting the color dipole cascade model with fixed order matrix elements, JHEP 05 (2002) 046 [hep-ph/0112284] [INSPIRE].

[5] N. Lavesson and L. Lönnblad, Merging parton showers and matrix elements: back to basics, JHEP 04 (2008) 085 [arXiv:0712.2966] [INSPIRE].

[6] J. Alwall et al., Comparative study of various algorithms for the merging of parton showers and matrix elements in hadronic collisions, Eur. Phys. J. C 53 (2008) 473 [arXiv:0706.2569] [INSPIRE].

[7] S. Höche, F. Krauss, S. Schumann and F. Siegert, QCD matrix elements and truncated showers, JHEP 05 (2009) 053 [arXiv:0903.1219] [INSPIRE]. 
[8] K. Hamilton, P. Richardson and J. Tully, A modified CKKW matrix element merging approach to angular-ordered parton showers, JHEP 11 (2009) 038 [arXiv:0905.3072] [INSPIRE].

[9] T. Carli, T. Gehrmann and S. Höche, Hadronic final states in deep-inelastic scattering with Sherpa, Eur. Phys. J. C 67 (2010) 73 [arXiv:0912.3715] [INSPIRE].

[10] L. Lönnblad and S. Prestel, Matching tree-level matrix elements with interleaved showers, JHEP 03 (2012) 019 [arXiv: 1109.4829] [INSPIRE].

[11] S. Frixione and B.R. Webber, Matching NLO QCD computations and parton shower simulations, JHEP 06 (2002) 029 [hep-ph/0204244] [INSPIRE].

[12] P. Nason, A new method for combining NLO QCD with shower Monte Carlo algorithms, JHEP 11 (2004) 040 [hep-ph/0409146] [INSPIRE].

[13] S. Frixione, P. Nason and C. Oleari, Matching NLO QCD computations with Parton Shower simulations: the POWHEG method, JHEP 11 (2007) 070 [arXiv:0709.2092] [INSPIRE].

[14] M.H. Seymour, Matrix element corrections to parton shower algorithms, Comput. Phys. Commun. 90 (1995) 95 [hep-ph/9410414] [INSPIRE].

[15] M.H. Seymour, A simple prescription for first order corrections to quark scattering and annihilation processes, Nucl. Phys. B 436 (1995) 443 [hep-ph/9410244] [INSPIRE].

[16] J. André and T. Sjöstrand, Matching of matrix elements and parton showers, Phys. Rev. D 57 (1998) 5767 [hep-ph/9708390] [INSPIRE].

[17] E. Norrbin and T. Sjöstrand, QCD radiation off heavy particles, Nucl. Phys. B 603 (2001) 297 [hep-ph/0010012] [INSPIRE].

[18] S. Alioli, K. Hamilton, P. Nason, C. Oleari and E. Re, Jet pair production in POWHEG, JHEP 04 (2011) 081 [arXiv: 1012.3380] [INSPIRE].

[19] S. Alioli, P. Nason, C. Oleari and E. Re, Vector boson plus one jet production in POWHEG, JHEP 01 (2011) 095 [arXiv: 1009.5594] [INSPIRE].

[20] S. Alioli, P. Nason, C. Oleari and E. Re, A general framework for implementing NLO calculations in shower Monte Carlo programs: the POWHEG BOX, JHEP 06 (2010) 043 [arXiv: 1002.2581] [INSPIRE].

[21] R. Frederix et al., aMC@NLO predictions for Wjj production at the Tevatron, JHEP 02 (2012) 048 [arXiv:1110.5502] [INSPIRE].

[22] ATLAS collaboration, G. Aad et al., Search for squarks and gluinos using final states with jets and missing transverse momentum with the ATLAS detector in $\sqrt{s}=7 \mathrm{TeV}$ proton-proton collisions, Phys. Lett. B 701 (2011) 186 [arXiv:1102.5290] [INSPIRE].

[23] ATLAS collaboration, G. Aad et al., Search for supersymmetry using final states with one lepton, jets and missing transverse momentum with the ATLAS detector in $\sqrt{s}=7 \mathrm{TeV} p p$, Phys. Rev. Lett. 106 (2011) 131802 [arXiv:1102.2357] [INSPIRE].

[24] CMS collaboration, S. Chatrchyan et al., Search for New Physics with a Mono-Jet and Missing Transverse Energy in pp Collisions at $\sqrt{s}=7$ TeV, Phys. Rev. Lett. 107 (2011) 201804 [arXiv: 1106 . 4775] [INSPIRE].

[25] CMS collaboration, S. Chatrchyan et al., Search for New Physics with Jets and Missing Transverse Momentum in pp collisions at $\sqrt{s}=7$ TeV, JHEP 08 (2011) 155 [arXiv: 1106.4503] [INSPIRE]. 
[26] CMS collaboration, S. Chatrchyan et al., Search for supersymmetry in pp collisions at $\sqrt{s}=7 \mathrm{TeV}$ in events with a single lepton, jets and missing transverse momentum, JHEP 08 (2011) 156 [arXiv:1107.1870] [INSPIRE].

[27] ATLAS collaboration, G. Aad et al., Measurement of the production cross section for $W$-bosons in association with jets in pp collisions at $\sqrt{s}=7$ TeV with the ATLAS detector, Phys. Lett. B 698 (2011) 325 [arXiv:1012.5382] [INSPIRE].

[28] ATLAS collaboration, G. Aad et al., A measurement of the ratio of the $W$ and $Z$ cross sections with exactly one associated jet in pp collisions at $\sqrt{s}=7$ TeV with ATLAS, Phys. Lett. B 708 (2012) 221 [arXiv:1108.4908] [INSPIRE].

[29] ATLAS collaboration, S. Ask, Measurement of the production cross section for $W$ - and Z-bosons in association with jets in ATLAS, arXiv:1106.2061 [INSPIRE].

[30] CMS collaboration, S. Chatrchyan et al., Measurement of the Polarization of W Bosons with Large Transverse Momenta in W+Jets Events at the LHC, Phys. Rev. Lett. 107 (2011) 021802 [arXiv: 1104.3829] [INSPIRE].

[31] CMS collaboration, C. Rogan, $W+$ jets and $Z+j e t s$ studies with the CMS detector at the CERN LHC, PoS (HCP2009) 096.

[32] J.M. Campbell and R.K. Ellis, Next-to-leading order corrections to $W^{+} 2$ jet and $Z^{+} 2$ jet production at hadron colliders, Phys. Rev. D 65 (2002) 113007 [hep-ph/0202176] [INSPIRE].

[33] R.K. Ellis, K. Melnikov and G. Zanderighi, $W+3$ jet production at the Tevatron, Phys. Rev. D 80 (2009) 094002 [arXiv:0906.1445] [INSPIRE].

[34] R.K. Ellis, K. Melnikov and G. Zanderighi, Generalized unitarity at work: first NLO QCD results for hadronic $W^{+}$3jet production, JHEP 04 (2009) 077 [arXiv:0901.4101] [INSPIRE].

[35] C. Berger et al., An Automated Implementation of On-Shell Methods for One-Loop Amplitudes, Phys. Rev. D 78 (2008) 036003 [arXiv:0803.4180] [INSPIRE].

[36] C. Berger et al., Precise Predictions for $W+3$ Jet Production at Hadron Colliders, Phys. Rev. Lett. 102 (2009) 222001 [arXiv:0902.2760] [InSPIRE].

[37] C. Berger et al., Next-to-Leading Order QCD Predictions for Z, $\gamma^{*}+3$-Jet Distributions at the Tevatron, Phys. Rev. D 82 (2010) 074002 [arXiv:1004.1659] [InSPIRE].

[38] C. Berger et al., Precise Predictions for $W+4$ Jet Production at the Large Hadron Collider, Phys. Rev. Lett. 106 (2011) 092001 [arXiv:1009.2338] [INSPIRE].

[39] H. Ita et al., Precise Predictions for Z+4 Jets at Hadron Colliders, Phys. Rev. D 85 (2012) 031501 [arXiv: 1108.2229] [INSPIRE].

[40] S. Hoche, F. Krauss, M. Schönherr and F. Siegert, Automating the POWHEG method in Sherpa, JHEP 04 (2011) 024 [arXiv: 1008.5399] [INSPIRE].

[41] S. Catani and M. Seymour, A general algorithm for calculating jet cross-sections in NLO QCD, Nucl. Phys. B 485 (1997) 291 [Erratum ibid. B 510 (1998) 503-504] [hep-ph/9605323] [INSPIRE].

[42] S. Catani, S. Dittmaier, M.H. Seymour and Z. Trócsányi, The dipole formalism for next-to-leading order QCD calculations with massive partons, Nucl. Phys. B 627 (2002) 189 [hep-ph/0201036] [INSPIRE].

[43] S. Frixione, Z. Kunszt and A. Signer, Three jet cross-sections to next-to-leading order, Nucl. Phys. B 467 (1996) 399 [hep-ph/9512328] [INSPIRE]. 
[44] S. Frixione, A general approach to jet cross-sections in QCD, Nucl. Phys. B 507 (1997) 295 [hep-ph/9706545] [INSPIRE].

[45] T. Plehn, D. Rainwater and P. Skands, Squark and gluino production with jets, Phys. Lett. B 645 (2007) 217 [hep-ph/0510144] [INSPIRE].

[46] R. Corke and T. Sjöstrand, Improved Parton Showers at Large Transverse Momenta, Eur. Phys. J. C 69 (2010) 1 [arXiv:1003.2384] [inSPIRE].

[47] K. Mikaelian, M. Samuel and D. Sahdev, The Magnetic Moment of Weak Bosons Produced

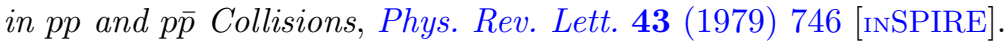

[48] C. Goebel, F. Halzen and J. Leveille, Angular zeros of Brown, Mikaelian, Sahdev and Samuel and the factorization of tree amplitudes in gauge theories, Phys. Rev. D 23 (1981) 2682 [INSPIRE].

[49] Z. Bern, J. Carrasco and H. Johansson, New Relations for Gauge-Theory Amplitudes, Phys. Rev. D 78 (2008) 085011 [arXiv: 0805.3993] [INSPIRE].

[50] S. Alioli, P. Nason, C. Oleari and E. Re, NLO vector-boson production matched with shower in POWHEG, JHEP 07 (2008) 060 [arXiv:0805.4802] [INSPIRE].

[51] S. Frixione, P. Nason and B.R. Webber, Matching NLO QCD and parton showers in heavy flavor production, JHEP 08 (2003) 007 [hep-ph/0305252] [INSPIRE].

[52] R. Frederix et al., $W$ and $Z / \gamma *$ boson production in association with a bottom-antibottom pair, JHEP 09 (2011) 061 [arXiv: 1106.6019] [INSPIRE].

[53] R. Frederix, private communication.

[54] Y.L. Dokshitzer, D. Diakonov and S. Troian, Hard Processes in Quantum Chromodynamics, Phys. Rept. 58 (1980) 269 [inSPIRE].

[55] A. Buckley et al., General-purpose event generators for LHC physics, Phys. Rept. 504 (2011) 145 [arXiv: 1101.2599$]$ [INSPIRE].

[56] S. Alioli, P. Nason, C. Oleari and E. Re, NLO Higgs boson production via gluon fusion matched with shower in POWHEG, JHEP 04 (2009) 002 [arXiv:0812.0578] [INSPIRE].

[57] S. Schumann and F. Krauss, A parton shower algorithm based on Catani-Seymour dipole factorisation, JHEP 03 (2008) 038 [arXiv:0709.1027] [INSPIRE].

[58] Z. Nagy, Next-to-leading order calculation of three jet observables in hadron hadron collision, Phys. Rev. D 68 (2003) 094002 [hep-ph/0307268] [INSPIRE].

[59] S. Höche, F. Krauss, M. Schönherr and F. Siegert, NLO matrix elements and truncated showers, JHEP 08 (2011) 123 [arXiv:1009.1127] [INSPIRE].

[60] S. Höche, S. Schumann and F. Siegert, Hard photon production and matrix-element parton-shower merging, Phys. Rev. D 81 (2010) 034026 [arXiv:0912.3501] [INSPIRE].

[61] S. Platzer and M. Sjodahl, The Sudakov veto algorithm reloaded, Eur. Phys. J. Plus 127 (2012) 26 [arXiv:1108.6180] [INSPIRE].

[62] T. Sjöstrand, S. Mrenna and P.Z. Skands, PYTHIA 6.4 Physics and Manual, JHEP 05 (2006) 026 [hep-ph/0603175] [INSPIRE].

[63] T. Gleisberg et al., SHERPA 1. alpha: a proof of concept version, JHEP 02 (2004) 056 [hep-ph/0311263] [INSPIRE]. 
[64] T. Gleisberg et al., Event generation with SHERPA 1.1, JHEP 02 (2009) 007 [arXiv:0811.4622] [INSPIRE].

[65] F. Krauss, R. Kuhn and G. Soff, $A M E G I C++$ 1.0: A matrix element generator in $\mathrm{C}++$, JHEP 02 (2002) 044 [hep-ph/0109036] [INSPIRE].

[66] T. Gleisberg and F. Krauss, Automating dipole subtraction for QCD NLO calculations, Eur. Phys. J. C 53 (2008) 501 [arXiv:0709.2881] [INSPIRE].

[67] P.M. Nadolsky et al., Implications of CTEQ global analysis for collider observables, Phys. Rev. D 78 (2008) 013004 [arXiv: 0802.0007] [INSPIRE].

[68] A. Buckley et al., Rivet user manual, arXiv:1003.0694 [INSPIRE].

[69] S. Catani, Y.L. Dokshitzer, M. Seymour and B. Webber, Longitudinally invariant $K_{\perp}$ clustering algorithms for hadron hadron collisions, Nucl. Phys. B 406 (1993) 187 [INSPIRE].

[70] S.D. Ellis and D.E. Soper, Successive combination jet algorithm for hadron collisions, Phys. Rev. D 48 (1993) 3160 [hep-ph/9305266] [INSPIRE].

[71] M. Cacciari and G.P. Salam, Dispelling the $N^{3}$ myth for the $k_{t}$ jet-finder, Phys. Lett. B 641 (2006) 57 [hep-ph/0512210] [INSPIRE].

[72] S. Dawson, Radiative corrections to Higgs boson production, Nucl. Phys. B 359 (1991) 283.

[73] A. Djouadi, M. Spira and P. Zerwas, Production of Higgs bosons in proton colliders: QCD corrections, Phys. Lett. B 264 (1991) 440 [INSPIRE].

[74] M.L. Mangano, M. Moretti, F. Piccinini and M. Treccani, Matching matrix elements and shower evolution for top-quark production in hadronic collisions, JHEP 01 (2007) 013 [hep-ph/0611129] [INSPIRE].

[75] J. Campbell, R. K. Ellis and C. Williams, MCFM - Monte Carlo for FeMtobarn processes, http://mcfm.fnal.gov.

[76] V. Ravindran, J. Smith and W. Van Neerven, Next-to-leading order QCD corrections to differential distributions of Higgs boson production in hadron hadron collisions, Nucl. Phys. B 634 (2002) 247 [hep-ph/0201114] [INSPIRE].

[77] J.-C. Winter, F. Krauss and G. Soff, A modified cluster hadronization model, Eur. Phys. J. C 36 (2004) 381 [hep-ph/0311085] [INSPIRE].

[78] F. Krauss et al., SHERPA's new hadronisation model, in preparation.

[79] T. Sjöstrand and M. van Zijl, A multiple interaction model for the event structure in hadron collisions, Phys. Rev. D 36 (1987) 2019 [INSPIRE].

[80] S. Alekhin et al., HERA and the LHC: A Workshop on the implications of HERA for LHC physics: Proceedings Part A, hep-ph/0601012 [INSPIRE].

[81] F. Krauss, T. Laubrich and F. Siegert, Simulation of hadron decays in SHERPA, in preparation.

[82] M. Schönherr and F. Krauss, Soft photon radiation in particle decays in SHERPA, JHEP 12 (2008) 018 [arXiv: 0810.5071] [INSPIRE].

[83] B. Andersson, G. Gustafson, G. Ingelman and T. Sjöstrand, Parton Fragmentation and String Dynamics, Phys. Rept. 97 (1983) 31 [inSPIRE].

[84] B. Andersson, Cambridge Monographs on Particle Physics, Nuclear Physics and Cosmology. Vol. 7: The Lund model, Cambridge University Press, Cambridge U.K. (1997). 
[85] CDF collaboration, T. Aaltonen et al., Measurement of inclusive jet cross-sections in $Z / \gamma^{*}(\rightarrow e e)+$ jets Production in $p \bar{p}$ Collisions at $\sqrt{s}=1.96$ TeV, Phys. Rev. Lett. 100 (2008) 102001 [arXiv:0711.3717] [INSPIRE].

[86] CDF collaboration, A. Abulencia et al., Measurement of the inclusive jet cross section in p $\bar{p}$ interactions at $\sqrt{s}=1.96 \mathrm{TeV}$ using a cone-based jet algorithm, Phys. Rev. D 74 (2006) 071103 [hep-ex/0512020] [INSPIRE].

[87] D0 collaboration, V. Abazov et al., Measurement of differential $Z / \gamma^{*}+$ jet $+X$ cross sections in pp collisions at $\sqrt{s}=1.96 \mathrm{TeV}$, Phys. Lett. B 669 (2008) 278 [arXiv:0808.1296] [INSPIRE].

[88] G.C. Blazey et al., Run II jet physics, hep-ex/0005012 [INSPIRE].

[89] D0 collaboration, V. Abazov et al., Measurements of differential cross sections of $Z / \gamma^{*}+$ jets $+X$ events in proton anti-proton collisions at $\sqrt{s}=1.96 \mathrm{TeV}$, Phys. Lett. B 678 (2009) 45 [arXiv: 0903.1748] [INSPIRE].

[90] D0 collaboration, V.M. Abazov et al., Measurement of $Z / \gamma^{*}+j e t+X$ angular distributions in $p \bar{p}$ collisions at $\sqrt{s}=1.96 \mathrm{TeV}$, Phys. Lett. B 682 (2010) 370 [arXiv:0907.4286] [inSPIRE].

[91] A. Banfi, G.P. Salam and G. Zanderighi, Resummed event shapes at hadron-hadron colliders, JHEP 08 (2004) 062 [hep-ph/0407287] [INSPIRE]. Erratum added online, 29 November 2004.

[92] G. Bozzi, S. Catani, D. de Florian and M. Grazzini, Transverse-momentum resummation and the spectrum of the Higgs boson at the LHC, Nucl. Phys. B 737 (2006) 73 [hep-ph/0508068] [INSPIRE].

[93] P. Nason and G. Ridolfi, A Positive-weight next-to-leading-order Monte Carlo for Z pair hadroproduction, JHEP 08 (2006) 077 [hep-ph/0606275] [INSPIRE].

[94] S. Catani, B. Webber and G. Marchesini, QCD coherent branching and semiinclusive processes at large $x$, Nucl. Phys. B 349 (1991) 635 [InSPIRE].

[95] M. Dasgupta and G. Salam, Resummation of the jet broadening in DIS, Eur. Phys. J. C 24 (2002) 213 [hep-ph/0110213] [INSPIRE].

[96] M. Dasgupta and G.P. Salam, Resummed event shape variables in DIS, JHEP 08 (2002) 032 [hep-ph/0208073] [INSPIRE].

[97] G. Bozzi, S. Catani, G. Ferrera, D. de Florian and M. Grazzini, Production of Drell-Yan lepton pairs in hadron collisions: Transverse-momentum resummation at next-to-next-to-leading logarithmic accuracy, Phys. Lett. B 696 (2011) 207 [arXiv: 1007.2351] [INSPIRE]. 